\title{
Potential Anti-Mycobacterium tuberculosis Activity of Plant Secondary Metabolites: Insight with Molecular Docking Interactions
}

\author{
Manu Kumar ${ }^{1}$ D, Sandeep Kumar Singh ${ }^{2}$, Prem Pratap Singh ${ }^{3}$, Vipin Kumar Singh ${ }^{3}$, Avinash Chandra Rai ${ }^{4}{ }^{10}$, \\ Akhileshwar Kumar Srivastava ${ }^{5} \mathbb{D}$, Livleen Shukla ${ }^{2} \mathbb{D}$, Mahipal Singh Kesawat ${ }^{6}$, Atul Kumar Jaiswal ${ }^{7}$, \\ Sang-Min Chung ${ }^{1, *}$ and Ajay Kumar ${ }^{8, *(\mathbb{D})}$
}

Citation: Kumar, M.; Singh, S.K.; Singh, P.P.; Singh, V.K.; Rai, A.C.; Srivastava, A.K.; Shukla, L.; Kesawat, M.S.; Kumar Jaiswal, A.; Chung, S.-M.; et al. Potential Anti-Mycobacterium tuberculosis Activity of Plant Secondary Metabolites: Insight with Molecular Docking Interactions. Antioxidants 2021, 10, 1990. https://doi.org/ 10.3390/antiox10121990

Academic Editor: Evangelos Zoidis

Received: 6 October 2021

Accepted: 9 December 2021

Published: 14 December 2021

Publisher's Note: MDPI stays neutral with regard to jurisdictional claims in published maps and institutional affiliations.

Copyright: (c) 2021 by the authors. Licensee MDPI, Basel, Switzerland. This article is an open access article distributed under the terms and conditions of the Creative Commons Attribution (CC BY) license (https:// creativecommons.org/licenses/by/ $4.0 /)$.
1 Department of Life Science, College of Life Science and Biotechnology, Dongguk University, Seoul 10326, Korea; manukumar007@gmail.com

2 Division of Microbiology, Indian Agricultural Research Institute, Pusa, New Delhi 110012, India; sandeepksingh015@gmail.com (S.K.S.); 1shukla65@gmail.com (L.S.)

3 Centre of Advance Study in Botany, Banaras Hindu University, Varanasi 221005, India; prempratapsingh31@gmail.com (P.P.S.); vipinks85@gmail.com (V.K.S.)

4 Institute of Plant Sciences, Agriculture Research Organization, Volcani Center, Rishon LeZion 7505101, Israel; avinashchandraraigene29@gmail.com

5 Plant Cell Biotechnology Department, CSIR-Central Food Technological Research Institute, Mysore 570020, India; akhileshwar.kumar2@gmail.com

6 Faculty of Agriculture, Sri Sri University, Cuttack 754006, India; mahipal.s@srisriuniversity.edu.in

7 School of Computational and Integrative Sciences, Jawaharlal Nehru University, New Delhi 110067, India; atulj91@gmail.com

8 Department of Postharvest Science, Agriculture Research Organization, Volcani Center, Rishon LeZion 7505101, Israel

* Correspondence: smchung@dongguk.edu (S.-M.C.); ajaykumar_bhu@yahoo.com (A.K.)

\begin{abstract}
Tuberculosis (TB) is a recurrent and progressive disease, with high mortality rates worldwide. The drug-resistance phenomenon of Mycobacterium tuberculosis is a major obstruction of allelopathy treatment. An adverse side effect of allelopathic treatment is that it causes serious health complications. The search for suitable alternatives of conventional regimens is needed, i.e., by considering medicinal plant secondary metabolites to explore anti-TB drugs, targeting the action site of M. tuberculosis. Nowadays, plant-derived secondary metabolites are widely known for their beneficial uses, i.e., as antioxidants, antimicrobial agents, and in the treatment of a wide range of chronic human diseases (e.g., tuberculosis), and are known to "thwart" disease virulence. In this regard, in silico studies can reveal the inhibitory potential of plant-derived secondary metabolites against Mycobacterium at the very early stage of infection. Computational approaches based on different algorithms could play a significant role in screening plant metabolites against disease virulence of tuberculosis for drug designing.
\end{abstract}

Keywords: plant secondary metabolites; antioxidant activity; drug discovery; multi-drug resistance (M.D.R.); molecular docking; tuberculosis

\section{Introduction}

Plants produce a diverse range of secondary metabolites (SMs), due to various physiological and metabolic processes, which, since ancient times, have served as "raw" material for enhancing the immune system and in the treatment of various human ailments. Currently, more than $75 \%$ of the global population, especially developing countries, rely on plant metabolites or natural products for their primary health treatments [1]. In addition, more than $25 \%$ of modern drugs and $60 \%$ of total anti-cancer drugs are derived from plant secondary metabolites, directly or indirectly [2,3]. The diverse range of functional 
groups of secondary metabolites offer opportunities to search or target the molecular sites of pathogens, which is an essential condition for drug discovery [4].

Tuberculosis (TB), one of the most contagious global diseases, is considered one of the top ten most lethal diseases worldwide [5]. TB, being a fatal infectious disease, has shown its devastating nature by infecting over 10.0 million ( 9.0-11.1 million) people worldwide in 2018. However, the burden of TB is increasing continuously, with approximately 5 to $>500$ new cases each year (per millions) of the global population; the global average is around 130 cases. Noteworthy, a higher severity of TB has been reported in developing countries. It is worth noting that the high impact of TB on the host immune system may increase the incidence of the disease [6]. There was a dramatic decline in the number of TB cases in the 1950-1970s, likely due to the discovery of an effective antimycobacterial agent viz. ethambutol, isoniazid, pyrazinamide, and rifampicin. However, presently, there is a massive upsurge in TB cases throughout the world due to the emergence of multi-drug resistance (MDR), extensively drug resistance (XDR), and total drug resistance (TDR) in mycobacteria [7]. The misuse of antimycobacterial drugs has led to the prevalence of these "outdriven" TB conditions (MDR and XDR). As per an estimate, in the global context, there was a substantial increment of up to 186,772 MDR-TB cases observed in 2018 compared to the previously reported 160,684 cases in 2017. The resistance developed in Mycobacterium tuberculosis $(\mathrm{Mtb})$ has caused inefficiency in the first-line and (some of the) second-line drugs commonly used for treatment. Additionally, popular second-line drugs, such as ethionamide, capreomycin, and kanamycin, employed to cure MDR/XDR-TB, have some safety concerns, with only a 50\% cure rate. While safer second-line drugs (e.g., ofloxacin and norfloxacin) have better efficiency, the drawback is they are expensive. Therefore, there is an urgent need to develop new, affordable, and effective anti-tuberculosis drugs with unique drug targets, multi-domain inhibitory effects, possessing fewer and negligible side effects [8].

Plants, being significant reservoirs of various biologically active compounds, play an essential role in curing several human diseases. Plant-derived phytochemicals have a long history of providing much-needed novel therapeutics $[9,10]$. The major portion of the globe, i.e., Africa, Asia, Latin America, and the Middle East, with $70-95 \%$ of the entire population, use traditional medicine for their primary healthcare needs [11]. In addition, several plant secondary metabolites, i.e., alkaloids, coumarins, flavonoids, polyphenols, terpenoids, triterpenoids, quinines, plumbagin, maritinone, 3,3'-biplumbagin, aloe-emodin, epigallocatechin, and umckalin, have been widely exploited for their broad-spectrum activities against various human diseases.

\section{Plant Secondary Metabolites as Antioxidant and Antimycobacterial Agents}

Secondary metabolites (SMs) produced by plants are defined as a different group of natural intermediary metabolic products that are not obligatorily required for the vegetative growth of plants [12]. These small molecules are derived mainly from the primary metabolites, in which some are nitrogen-containing alkaloids (e.g., amino acids, amines, cyanogenic glycosides, and glucosinolates), non-nitrogen compounds polyphenols, terpenoids, flavonoids, steroids, lignin, and tannins [13]. Since ancient times, plant extracts have been used as (an easy source of) antibiotics/antioxidants and applied as crude/extract against bacterial or fungal infections, with minimal side effects [14]. Out of all the plant-synthesized metabolites, alkaloids and polyphenols have potent antimicrobial and antioxidant properties. Alkaloids have a possible role in the development of antibiotics, whereas plenty of polyphenols provide a wide range of antioxidant properties that eventually establish the basis of antimicrobial activity [15]. Extreme environmental changes and various physiological or metabolic processes of the body can generate free radicals, which are continuously neutralized by antioxidant molecules. The optimum requirements of antioxidant molecules is required for the complete neutralization of free radicals. The excess accumulation of free radicals provokes cellular damage and can cause several fatal diseases, including cancer, diabetes, Alzheimer's disease, and aging [16,17]. 
SMs, such as polyphenols, have great potential in neutralizing free radicals, and are excellent antioxidants molecules [17]. The polyphenols derived from plant crude was shown to neutralize ROS free radicals [18]. Polyphenols scavenge the singlet and triplet oxygengenerated free radicals to provide hydrogen as a donor molecule [16]. Several studies have proved that crude extracts of different medicinal plants have high antioxidant and antimicrobial potential.

Crude extract of flowers Wendlandia thyrsoidea, Olea dioica, Lagerstroemia speciosa, and Bombax malabaricum species showed the great potential of antioxidant and antimicrobial activity, in regard to the presence of phenolic and flavonoids [19]. Ziziphus lotus and Ziziphus mauritiana leave fruit and seed extract with higher phenolic flavonoids and tannins, which show tremendous antioxidant capacity and have been successfully used against different bacterial strains [20]. Similarly, stem bark extract of Crateva religiosa showed antimicrobial and antifungal activity due to the presence of phenolic phytochemicals. Therefore, it can be formulated for drug discovery in the future for pharmaceutical industries [21]. Natural bioactive compounds present in Nepeta trachonitica showed high phenolic content, and antimicrobial as well as antioxidant activity. HPLC-MS/MS data reveal that these medicinal plants have high phenolic compounds and could be a promising source of nutraceutical and drug industries [22]. Colorimetric, chromatographic, and spectrophotometric assays revealed that $P$. granatum (pomegranate) leaf extract showed a high content of total phenols, ortho-diphenols, tannins, and antioxidant capacity, making pomegranate leaf extract a valued plant source of accepted bioactive molecules for emerging beneficial food-pharma ingredients [23].

SMs are highly economically valuable products because of the current clinical use of drug plants. They have been used extensively as a drug, flavors, fragrances, etc. Plants synthesize a considerable number of phenols and derivatives as aromatic substances [24]. Thousands of terpenoids are used extensively to produce drugs synthesized from the fivecarbon precursor isopentyl diphosphate. However, around 12,000 alkaloids with nitrogen atoms are biosynthesized from amino acids. Alkaloids are used as salts in medicine, such as quinine, vinblastine, and reserpine [25,26]. Currently, alkaloids are used for analgesics, anti-cancer agents, muscle relaxants, antibiotics, and sedatives.

Furthermore, around 8000 phenolic compounds are synthesized from the malonate/ acetate or shikimic acid pathway [27]. Studies reveal that phenols have antimicrobial, antiviral, and anti-inflammatory actions [28-30]. During oxidative damage, phenolic compounds act as antioxidants and protect against the damage of cells from oxidative stress. Phenolic compounds have neuroprotective, fungicidal, and bactericidal activities [31-33]. Moreover, it has been well documented that phenolic compounds have anti-atherosclerosis and anti-cancer activity [34,35].

Plant products are virtual repositories for the development of new drugs, with minimal side effects on humans. The extensive array of phytochemicals possessing antioxidant activities is are required for the therapeutic activity of plant products against human diseases, including tuberculosis [36,37]. The aqueous and ethanolic extract of Piper sarmentosum harboring antioxidant activity is reported to exhibit antitubercular activity [38]. The antioxidant activity determined for different parts of the selected plant using DPPH and beta-carotene linoleic acid assay displayed substantial variations. Alcoholic extract was observed to have better antioxidant potential in comparison to aqueous extract. Recently, essential oil, and a major component, viridiflorol, derived from Allophylus edulis, have demonstrated antioxidant and anti-tuberculosis activity [39]. The investigation used the DPPH and ABTS assay to measure antioxidant activity of significant components and essential oils. The radical scavenging activity percentage of essential oil and viridiflorol as measured by ABTS was $44.33 \%$ and $57.55 \%$, respectively. The antioxidant value determined by the DPPH assay, and represented as $\mathrm{IC}_{50}$, was $82.9 \%$ and $74.7 \%$, respectively, suggesting moderate activity compared to the reference materials (butylated hydroxytoluene and ascorbic acid). The antioxidant activity antitubercular activities of plants, including Globularia alypum, Acacia catechu, Ailanthus excelsa, Aegle marmelos, Andrographis 
paniculata, Datura metel, and Aegiceras corniculatum, have also been registered by different researchers globally [40-42], indicating the potential opportunities of huge plant diversity in treating life-threatening diseases (e.g., tuberculosis). However, the antioxidant activity of plants varies considerably, depending on the nature of phytochemicals, the method of extraction, climatic conditions, methods of measuring antioxidant activity, and the plant parts selected. One of the major limitations of using plant products having antioxidant activity for treating tuberculosis may be the restricted synthesis of the target compound by the plant itself. However, such hurdles can be resolved to some extent by using modern genetic engineering approaches to direct the compound synthesis in the desired quantity.

Conventional methods of metabolites screening, such as high throughput screening (HTS) and virtual high throughput screening (vHTS), have been used to speed up the drug discovery for time-efficient identification of cost-effective novel and selective metabolites. However, HTS explored bulky hydrophobic metabolites poorly suited to chemical modification, requiring higher costs and time. Few vHTS success stories have been explained, identifying plant metabolites against specific virulent proteins, such as Dengue virus proteins [43]. Docking is the greatest tool of bioinformatics employed to determine the binding pose and binding score. Docking has been considered a "leader" in the present era, performing a range of identifications of plant metabolites to candidate leads for drug development [44]. The perfect binding of the compound provides the best scoring function that "implicates" in exploring the novel candidate complex and, hence, reduces the efforts needed in experimental work. The advancements in computational technology have "escorted" the synthesis of nature-based drugs, such as dasatinib and imatinib (approved by the FDA) [45]. Network pharmacology network procedures have increased the binding associations between ligands and their targets [46]. Docking has become an important methodological feature in computer added drug design (CADD). Docking is vital in determining the novel ligand from a medicinal plant for targeted proteins for structure-based drug designs [47]. Hence, docking will help increase crucial knowledge about the therapeutic potential of plant metabolites [48].

As per the literature review, several reports and studies show the potential of natural products as antimycobacterial agents. Mitscher and Baker [49] accounted for various plant-derived compounds as potential antitubercular agents. Gautam et al. [50] reported more than 200 plants having potent anti-tuberculosis activity, signifying the potential of natural products to remedy life-threatening diseases, such as TB. Drug discoveries based on computational approaches provide novel alternative tools to reduce the expensive and tedious identification of potential drug leads. Ligand-based computational screening has been used to characterize and identify new potential inhibitors and drug repurposing [44]. Miryalaa et al. [51] worked on 15 natural compounds to explore their anti-TB properties, employing in silico methods, and compared their potential with conventional drugs against $\mathrm{TB}$ and their respective protein targets. Interactive studies showed that glycyrrhizin, swertiamarin, and laccaic acid exhibit better binding affinity than conventional anti-TB drugs. Hence, glycyrrhizin, laccaic acid, and swertiamarin could be used to develop multitarget alternative drug candidates. Inhibition of important enzymes responsible for vital cellular functions, hence survivability of mycobacteria in the host system, is just one critical strategy used to deal with the (continuously rising) global TB incidents. In the present study, five plant secondary metabolites (alliin, aloin, octyl- $\beta$-d-glucopyranoside, oleanolic acid, and phytol) were evaluated against two standard front line anti-TB drugs, isoniazid (ISN) and ethambutol (EMB), to decipher their potential anti-tuberculosis efficacy, targeting four of the mycobacterial receptor proteins/enzymes (arabinosyltransferase $C$, protein kinase A, glutamine synthetase, and proteasomal ATPase) via in silico approaches.

\section{Current Status and Severity of Tuberculosis}

Geographical data over the TB epidemic showed South-East Asia as the most affected part of the world, with $44 \%$ of the total cases alone in this region. Further, eight countries accounted for two-thirds of the global total: India (26\%), Indonesia (8.5\%), China (8.4\%), 
the Philippines (6.0\%), Pakistan (5.7\%), Nigeria (4.4\%), Bangladesh (3.6\%), and South Africa (3.6\%). The other 22 other countries on WHO's list of 30 high TB-burdened countries accounted for $21 \%$ of the global total (Global Tuberculosis Report 2020 (released on 14 October 2020; https:/ /www.who.int/publications/i/item/9789240013131). In addition, on the list of drug-resistant TB countries, India again handled the largest disease burden $(130,000$ new cases in 2018) in sharing with countries, i.e., China and the Russian Federation. TB statistics in India reveal the concerning burden status. There were 449,000 deaths caused by in 2018, including $2.16 \%$ of deaths of people with HIV. Among the causes of deaths in all age groups, TB was in the top five. In accordance with the previous year's data (2000-2018), the disease incidence and disease-death rates declined in India because of the success of various treatment programs. In these TB treatment programs, the prescribed medicines cover $81 \%$ of the treatment success rate.

Nevertheless, these figures are not enough to provide relief because of the emergence of the toxic effects of synthetic drugs. Furthermore, there seems to be an increasing trend of side effect reports regarding the drug regimens administered to treat TB $[52,53]$. Therefore, there is an urgent need to search for safer alternatives for the treatment of TB, so that patient safety can be ensured.

\section{Management of MDR-Mtb: A Herbal Approach}

Researchers are exploring novel antimycobacterial compounds that have lesser side effects due to the development of multidrug-resistant TB and severe side effects of the synthetic drugs used for treatment. A list of side effects caused by various synthetic drugs is described in Table 1, along with generic names of the medicine.

Table 1. The list of side effects caused by various synthetic drugs, along with generic names.

\begin{tabular}{|c|c|c|c|c|}
\hline Generic Names & Medicinal Compound & Side Effects & Mode of Action & References \\
\hline $\begin{array}{l}\text { Isoniazid, isoniazide, azuren, } \\
\text { INH, L 1945, Mybasan, } \\
\text { neumadin, RP 5015, tubomel, } \\
\text { vazadrine, isoniazidum }\end{array}$ & $\begin{array}{c}\text { Isonicotinic acid } \\
\text { hydrazide (isoniazid) }\end{array}$ & $\begin{array}{l}\text { Hepatotoxic (hepatitis, } \\
\text { nausea, vomiting, and } \\
\text { decreased appetite) }\end{array}$ & $\begin{array}{l}\text { Suppresses the } \\
\text { multiplication of } \\
\text { mycobacteria }\end{array}$ & [54] \\
\hline $\begin{array}{l}\text { Streptomicina, streptomycin, } \\
\text { streptomycine, strepidin- } 4-\alpha- \\
\text { streptobiosaminosid, } \\
\text { streptomycin sulfate, } \\
\text { streptomycini sulfas, } \\
\text { streptomycinsulfat }\end{array}$ & Streptomycin & Ototoxicity & $\begin{array}{l}\text { Inhibition of protein } \\
\text { synthesis of mycobacteria in } \\
\text { the ribosome }\end{array}$ & \\
\hline $\begin{array}{l}\text { Ethionamide, TH 1314, } \\
\text { aethionamidum, Bayer 5312, } \\
\text { etionizina, ETP, ethionamidum }\end{array}$ & Ethionamide & $\begin{array}{l}\text { Hepatitis, depression, } \\
\text { hypersensitivity }\end{array}$ & $\begin{array}{l}\text { A prodrug that is activated } \\
\text { by the enzyme ethA, a } \\
\text { mono-oxygenase in } \\
\text { Mycobacterium tuberculosis; } \\
\text { binds NAD+ to form an } \\
\text { adduct that inhibits InhA in } \\
\text { the same way as isoniazid }\end{array}$ & [55] \\
\hline $\begin{array}{l}\text { Cycloserine, lilly 106-7, MK 65, } \\
\text { PA 94, Ro 1-9213, SC 49088, } \\
\text { cicloserina, cycloserinum }\end{array}$ & Cycloserine & Psychosis, rashes & $\begin{array}{c}\text { Cycloserine is a } \\
\text { broad-spectrum antibiotic } \\
\text { with only moderate anti-TB } \\
\text { activity. It inhibits cell wall } \\
\text { synthesis. The MIC of } \\
\text { cycloserine in the Bactec } \\
460-\mathrm{TB} \text { system is } \\
25-75 \mu \mathrm{g} / \mathrm{mL}\end{array}$ & \\
\hline
\end{tabular}


Table 1. Cont

\begin{tabular}{|c|c|c|c|c|}
\hline Generic Names & Medicinal Compound & Side Effects & Mode of Action & References \\
\hline $\begin{array}{l}\text { Capreomycin sulfate, } \\
\text { capreomycin, CAM, } \\
\text { capromycin, L } 29275\end{array}$ & Capreomycin & $\begin{array}{l}\text { Deafness, vestibular } \\
\text { toxicity }\end{array}$ & $\begin{array}{c}\text { Inhibit protein synthesis by } \\
\text { binding to the } 70 S \text { ribosomal } \\
\text { unit }\end{array}$ & \\
\hline $\begin{array}{l}\text { Kanamicina, kanamycin, } \\
\text { kanamycine, kanamycin } \\
\text { monosulfate, kanamycin } \\
\text { sulfate, kanamycin acid sulfate, } \\
\text { kanamycin monosulfate, } \\
\text { kanamycinmonosulfat }\end{array}$ & Kanamycin & Deafness, nephrotoxic & $\begin{array}{l}\text { Inhibits protein synthesis by } \\
\text { tightly binding to the } \\
\text { conserved A site of } 16 \mathrm{~S} \\
\text { rRNA in the } 30 \mathrm{~S} \text { ribosomal } \\
\text { subunit }\end{array}$ & {$[55]$} \\
\hline Rifampicin & Rifampicin & $\begin{array}{l}\text { Hepatotoxic, } \\
\text { interaction with other } \\
\text { drugs, a potent inducer } \\
\text { of microsomal enzymes }\end{array}$ & $\begin{array}{c}\text { Inhibits bacterial } \\
\text { DNA-dependent RNA } \\
\text { synthesis by inhibiting } \\
\text { bacterial DNA-dependent } \\
\text { RNA polymerase }\end{array}$ & \\
\hline $\begin{array}{l}\text { Pirazinamide, pyrazinamide, } \\
\text { pyrazinecarboxamide, } \\
\text { pyrazinoic acid amide, } \\
\text { pyrizinamide, pyrazinamidum }\end{array}$ & Pyrazinamide & $\begin{array}{c}\text { Hepatitis, } \\
\text { Hyperuricemia, } \\
\text { arthralgia, arthritis }\end{array}$ & $\begin{array}{c}\text { It diffuses into the } \\
\text { granuloma of } M \text {. tuberculosis, } \\
\text { where the tuberculosis } \\
\text { enzyme pyrazinamidase } \\
\text { converts pyrazinamide to the } \\
\text { active form of pyrazinoic } \\
\text { acid }\end{array}$ & {$[56]$} \\
\hline $\begin{array}{l}\text { Ethambutol, ethambutolo, } \\
\text { ethambutol hydrochloride, CL } \\
\text { 40881, ethambutol } \\
\text { hydrochloride, } \\
\text { ethambutoldihydrochlorid, } \\
\text { ethambutoli hydrochloridum }\end{array}$ & Ethambutol & Optic neuritis & $\begin{array}{l}\text { It works by obstructing the } \\
\text { formation of the cell wall. } \\
\text { Mycolic acids attach to the } \\
5^{\prime} \text {-hydroxyl groups of } \\
\text { D-arabinose residues of } \\
\text { arabinogalactan and form } \\
\text { mycolylarabinogalactan- } \\
\text { peptidoglycan complex in } \\
\text { the cell wall }\end{array}$ & \\
\hline $\begin{array}{c}\text { Protionamide, PTH, PTP, RP, } \\
\text { protionamidum, } \\
\text { prothionamide }\end{array}$ & Prothionamide & $\begin{array}{l}\text { Hepatotoxic, } \\
\text { hypersensitivity, } \\
\text { idiosyncrasy }\end{array}$ & $\begin{array}{c}\text { It is activated by } \\
\text { mono-oxygenase (EthA), } \\
\text { forms covalent adducts with } \\
\text { nicotinamide adenine } \\
\text { dinucleotide (NAD), and } \\
\text { inhibits InhA, leading to } \\
\text { blocking of the mycolic acid } \\
\text { pathway }\end{array}$ & [57] \\
\hline $\begin{array}{l}\text { P.A.S., Para-aminosalicylic } \\
\text { acid, pasalicylum, } \\
\text { aminosalicylic acid, } \\
\text { aminosalicylate sodium, } \\
\text { para-aminosalicylsaures } \\
\text { natrium-2-wasser, parasal } \\
\text { sodium, sodium } \\
\text { para-aminosalicylate, natrii } \\
\text { aminosalicylas dihydricus, } \\
\text { sodium aminosalicylate } \\
\text { dihydrate }\end{array}$ & $\begin{array}{l}\text { Para-aminosalicylic } \\
\text { acid }\end{array}$ & $\begin{array}{l}\text { Hepatotoxic, } \\
\text { hypersensitivity, } \\
\text { idiosyncrasy }\end{array}$ & $\begin{array}{l}\text { It targets dihydrofolate } \\
\text { reductase (DHFR); it is } \\
\text { incorporated into the folate } \\
\text { pathway by two enzymes, } \\
\text { dihydropteroate synthase } \\
\text { (DHPS) and dihydrofolate } \\
\text { synthase (DHFS) to produce } \\
\text { a hydroxyl dihydrofolate } \\
\text { compound that inhibits } \\
\text { DHFR, and subsequently } \\
\text { blocks the folate pathway }\end{array}$ & \\
\hline
\end{tabular}

Various plants and their metabolites elicit the desired effects against the virulent disease factors under in vivo and in vitro conditions. Plant-derived chemicals proved to be the better mycobacteria-inhibitory substances, with less (or no) side effects, ensuring the fast recovery of the patients. Jimenez-Arellanes et al. [58] evaluated the antimycotic activity of aqueous, methanolic, and n-hexane extract of 22 different plants against $M$. tuberculosis 
$\mathrm{H} 37 \mathrm{Rv}$ and $M$. avium at concentrations ranging from 50 to $200 \mu \mathrm{g} / \mathrm{mL}$. In a case study, Fauziyah et al. [59] checked the efficiency of the combined effects of anti-tuberculosis drugs and ethanolic extract of some specific medicinal plants against multi-drug resistant Mtb isolates. They concluded that a combination of plant extracts and rifampicin achieved better effects against the rifampicin/streptomycin-resistant strain. However, they also observed the antagonistic effects with streptomycin, ethambutol, and isoniazid. Nowadays, plant extracts and their metabolites are broadly used to treat MDR in several other human pathogens, viz. Staphylococcus aureus (wound and bloodstream infections), Escherichia coli (causing urinary tract infections), and Klebsiella pneumoniae (causing pneumonia, urinary tract, and bloodstream infections). It is estimated that between 2005 and 2015, a total of 110 purified compounds and 60 plant extracts were obtained from 112 different plants having potential effectiveness against MDR pathogens [60]. Details of the plants showing antimycobacterial activity are listed in Table 2.

Table 2. List of reported plant extracts with anti-tuberculosis activities against different $M$. tuberculosis isolates.

\begin{tabular}{|c|c|c|c|c|}
\hline $\begin{array}{c}\text { Plant } \\
\text { (Bioactive Compound) }\end{array}$ & Extract & Mtb & MIC & References \\
\hline $\begin{array}{c}\text { Lantana hispida } \\
\text { (-acetoxy-22-(2'-methyl-2Z- } \\
\text { butenyloxy)-12-oleanen-28-oic acid, } \\
\text { hydroxy-22 } \beta \text {-(2'-methyl-2Z- } \\
\text { butenoyloxy)-12-oleanen-28-oic acid } \\
\text { and oleanolic acid) }\end{array}$ & Hexane extract & $\begin{array}{c}\text { Mycobacterium } \\
\text { tuberculosis strain } \\
\text { H37Rv }\end{array}$ & $\begin{array}{l}50,50 \text { and } 25 \mu \mathrm{g} / \mathrm{mL} \\
\text { respectively }\end{array}$ & {$[61]$} \\
\hline Taxus baccata & $\begin{array}{l}\text { Chloroform extract of } \\
\text { heartwood and } \\
\text { ethanolic extract of } \\
\text { leaves }\end{array}$ & $\begin{array}{l}\text { M. tuberculosis strain } \\
\text { H37Ra }\end{array}$ & $200 \mu \mathrm{g} / \mathrm{mL}$ & {$[62]$} \\
\hline $\begin{array}{c}\text { Adhatoda vasica (2-acetyl } \\
\text { benzylamine and vasicine acetate) }\end{array}$ & Hexane extract & Mtb & $\begin{array}{l}200 \text { and } 50 \mu \mathrm{g} / \mathrm{mL}, \\
\text { respectively }\end{array}$ & {$[63]$} \\
\hline Terminalia phanerophlebia & $\begin{array}{c}\text { Ethanolic extract of } \\
\text { leaves }\end{array}$ & M. tuberculosis H37Ra & $390 \mu \mathrm{g} / \mathrm{mL}$ & {$[64]$} \\
\hline Opuntia ficus-indica & $\begin{array}{l}\text { Methanolic extract of } \\
\text { the plant (summer } \\
\text { season) }\end{array}$ & $\begin{array}{l}\text { M. tuberculosis strain } \\
\text { H37Rv (ATCC 27294) }\end{array}$ & $50 \mu \mathrm{g} / \mathrm{mL}$ & [65] \\
\hline Angiopteris evecta & $\begin{array}{c}\text { Methanolic extract of } \\
\text { leaves }\end{array}$ & \multirow[b]{2}{*}{ M. tuberculosis H37Rv } & $400 \mu \mathrm{g} / \mathrm{mL}$ & \multirow[b]{2}{*}[66]{} \\
\hline $\begin{array}{c}\text { Costus speciosus, Piper sarmentosum, } \\
\text { Pluchea indica, Pluchea indica, and } \\
\text { Tabernaemontana coronaria }\end{array}$ & Methanolic extract & & $800 \mu \mathrm{g} / \mathrm{mL}$ & \\
\hline Zanthoxylum capense (Decarine) & $\begin{array}{l}\text { Methanolic extract of } \\
\text { roots }\end{array}$ & $\begin{array}{l}\text { M. tuberculosis H37Ra } \\
\text { (ATCC 25177) and M. } \\
\text { tuberculosis H37Rv } \\
\text { (ATCC 27294) }\end{array}$ & $1.6 \mu \mathrm{g} / \mathrm{mL}$ & {$[67]$} \\
\hline Helichrysum devium & \multirow{3}{*}{ Methanolic extract } & \multirow{3}{*}{ M. tuberculosis H37Rv } & $50 \mu \mathrm{g} / \mathrm{mL}$ & \multirow{3}{*}[68]{} \\
\hline H. melaleucum & & & $100 \mu \mathrm{g} / \mathrm{mL}$ & \\
\hline H. obconicum & & & $200 \mu \mathrm{g} / \mathrm{mL}$ & \\
\hline $\begin{array}{l}\text { Artemisia capillaris (hydroquinone } \\
\text { and ursolic acid) }\end{array}$ & Methanolic extract & $\begin{array}{l}\text { M. tuberculosis strain } \\
\text { H37Rv and two clinical } \\
\text { isolates (resistant and } \\
\text { sensitive) }\end{array}$ & $\begin{array}{c}12.5 \mu \mathrm{g} / \mathrm{mL} \text { against } \\
\text { sensitive strains of } \mathrm{Mtb} \\
\text { while a range of } 12.5 \text { to } \\
25 \mu \mathrm{g} / \mathrm{mL} \text { against the } \\
\text { resistant strains }\end{array}$ & {$[69]$} \\
\hline
\end{tabular}


Table 2. Cont.

\begin{tabular}{|c|c|c|c|c|}
\hline $\begin{array}{c}\text { Plant } \\
\text { (Bioactive Compound) }\end{array}$ & Extract & Mtb & MIC & References \\
\hline Curtisia dentata & $\begin{array}{c}\text { Methanolic extract of } \\
\text { leaves }\end{array}$ & \multirow{2}{*}{$\begin{array}{l}\text { M. tuberculosis H37RV } \\
\text { (ATCC 27294) }\end{array}$} & $22.2 \mu \mathrm{g} / \mathrm{mL}$ & \multirow{2}{*}[70]{} \\
\hline Curtisia dentata (ursolic acid acetate) & Ethanolic extract & & $3.4 \mu \mathrm{g} / \mathrm{mL}$ & \\
\hline Aristolochia taliscana (Licarin A) & Hexane extract & $\begin{array}{l}\text { M. tuberculosis strains: } \\
\text { H37Rv, four } \\
\text { mono-resistant } \mathrm{H} 37 \mathrm{Rv} \\
\text { variants and } 12 \text { clinical } \\
\text { MDR isolates }\end{array}$ & $3.12-12.5 \mu \mathrm{g} / \mathrm{mL}$ & {$[71]$} \\
\hline Excoecaria agallocha & Methanolic extract & $\begin{array}{l}\text { M. tuberculosis } \mathrm{H} 37 \mathrm{Rv} \\
\text { and two clinical isolates } \\
\text { of Mtb }\end{array}$ & $\begin{array}{c}88.95 \% \text { of } \\
\text { antimycobacterial } \\
\text { activity against } M \text {. } \\
\text { tuberculosis } \mathrm{H} 37 \mathrm{Rv} \\
\text { while } 70.02 \% \text { and } \\
82.54 \% \text { for other two } \\
\text { isolates at } 500 \mu \mathrm{g} / \mathrm{mL} \\
\text { concentration }\end{array}$ & {$[72]$} \\
\hline Lantana camara & $\begin{array}{l}\text { Chloroform and } \\
\text { methanol extracts of } \\
\text { leaves }\end{array}$ & $\begin{array}{c}\text { Mycobacterium } \\
\text { tuberculosis H37Rv, } \\
\text { rifampicin-resistant } \\
\text { TMC-331 and a } \\
\text { non-resistant } \\
\text { wildstrain }(28-25271\end{array}$ & $\begin{array}{c}5.0 \mathrm{mg} / \mathrm{mL} \text { to } 50.0 \\
\mathrm{mg} / \mathrm{mL}\end{array}$ & [73] \\
\hline Solanum toroum Sw. & $\begin{array}{l}\text { hydro-ethanolic } \\
\text { extracts }\end{array}$ & $\begin{array}{l}\text { Mycobacterium } \\
\text { tuberculosis H37Ra }\end{array}$ & $156.3 \mu \mathrm{g} / \mathrm{mL}$ & [74] \\
\hline Alpinia galanga L. Willd. & $\begin{array}{l}\text { Acetone, aqueous and } \\
\text { ethanolic extracts of } \\
\text { rhizomes }\end{array}$ & $\begin{array}{c}\text { Mycobacterium } \\
\text { tuberculosis (M.tb) } \\
\text { H37Rv }\end{array}$ & $50-100 \mu \mathrm{g} / \mathrm{mL}$ & [75] \\
\hline $\begin{array}{l}\text { Lantana camara L., } \\
\text { Euphorbia hirta L., Mukia } \\
\text { maderaspatana (L.) M. Roem, and } \\
\text { Abutilon indicum L. }\end{array}$ & $\begin{array}{c}\text { Methanolic crude } \\
\text { extracts }\end{array}$ & $\begin{array}{c}\text { Mycobacterium } \\
\text { tuberculosis }(\mathrm{Mtb}) \text { and } \\
\text { Mtb H37Rv }\end{array}$ & $400-1600 \mu \mathrm{g} / \mathrm{mL}$ & {$[76]$} \\
\hline Artemisia annua & \multirow{2}{*}{$\begin{array}{l}\text { Dichloromethane } \\
\text { extracts }\end{array}$} & \multirow{2}{*}{$\begin{array}{c}\text { Mycobacterium } \\
\text { tuberculosis }(\mathrm{Mtb}), \\
\text { Mycobacterium abscessus }\end{array}$} & $37.5 \mu \mathrm{g} / \mathrm{mL}$ & \multirow{2}{*}{ [77] } \\
\hline and $A$. afra & & & $<1.3 \mu \mathrm{g} / \mathrm{mL}$ & \\
\hline Zingiber officinale & $\begin{array}{c}\text { Hydroethanolic extract } \\
\text { of rhizomes }\end{array}$ & \multirow{3}{*}{$\begin{array}{l}\text { M. tuberculosis H37Rv } \\
\text { (ATCC 27294) }\end{array}$} & $1250 \mu \mathrm{g} / \mathrm{mL}$ & \multirow{3}{*}{ [78] } \\
\hline Vitellaria paradoxa & \multirow{2}{*}{$\begin{array}{l}\text { Hydroethanolic extract } \\
\text { of bark }\end{array}$} & & $78.13 \mu \mathrm{g} / \mathrm{mL}$ & \\
\hline Alstonia boonei & & & $156 \mu \mathrm{g} / \mathrm{mL}$ & \\
\hline Musa spp. AAB, cv. "Manzano" & $\begin{array}{l}\text { n-hexane extract and } \\
\text { ethyl acetate extract }\end{array}$ & $\begin{array}{l}\text { Mycobacterium } \\
\text { tuberculosis }\end{array}$ & $\begin{array}{c}12.5 \text { and } \\
6.251 \mathrm{~g} / \mathrm{mL}\end{array}$ & [79] \\
\hline Trixis angustifolia & Hexane extract & $\begin{array}{l}\text { Mycobacterium } \\
\text { tuberculosis H37Rv }\end{array}$ & $12.5-25.0 \mu \mathrm{g} / \mathrm{mL}$ & [80] \\
\hline Acacia farnesiana & $\begin{array}{l}\text { hexane, chloroform and } \\
\text { methanolic } \\
\text { extracts }\end{array}$ & $\begin{array}{c}\text { Mycobacterium } \\
\text { tuberculosis H37Rv and } \\
\text { G122 }\end{array}$ & $100-200 \mu \mathrm{g} / \mathrm{mL}$ & [81] \\
\hline Pterolobium stellatum (Forssk) & \multirow{3}{*}{ Chloroform extracts } & \multirow{3}{*}{$\begin{array}{c}\text { M. tuberculosis strain } \\
\text { H37RV }\end{array}$} & $0.312 \mathrm{mg} / \mathrm{mL}$ & \multirow{3}{*}{ [82] } \\
\hline Persea americana Mill L. & & & $2.5 \mathrm{mg} / \mathrm{mL}$ & \\
\hline Otostegia integrifolia Benth L. & & & $0.312 \mathrm{mg} / \mathrm{mL}$ & \\
\hline
\end{tabular}


Table 2. Cont.

\begin{tabular}{|c|c|c|c|c|}
\hline $\begin{array}{c}\text { Plant } \\
\text { (Bioactive Compound) }\end{array}$ & Extract & Mtb & MIC & References \\
\hline $\begin{array}{l}\text { Aegle marmelos L, Glycyrrhiza glabra } \\
\text { L, Lawsonia inermis L, Piper nigrum L, } \\
\text { and Syzygium aromaticum L. }\end{array}$ & Methanolic extract & $\begin{array}{c}\text { M. tuberculosis strain } \\
\text { H37RV }\end{array}$ & 0.8 to $100 \mu \mathrm{g} / \mathrm{mL}$ & [83] \\
\hline $\begin{array}{c}\text { Boswellia serrata Roxb. ex, Datura } \\
\text { stramonium L and Lavandula stoechas } \\
\text { L. }\end{array}$ & Ethanolic extracts & $\begin{array}{c}\text { M. tuberculosis strain } \\
\text { H37RV }\end{array}$ & 125 to $250 \mu \mathrm{g} / \mathrm{mL}$ & {$[84]$} \\
\hline Pinus merkusii & Ethanolic extract & $\begin{array}{l}\text { Mycobacterium } \\
\text { tuberculosis H37Rv }\end{array}$ & $1000 \mu \mathrm{g} / \mathrm{mL}$ & [85] \\
\hline Dendrophthoe falcata $\mathrm{L}$. & \multirow{2}{*}{$\begin{array}{l}\text { Ethanol water and } \\
\text { methanol: water } \\
\text { extracts }\end{array}$} & \multirow{2}{*}{$\begin{array}{c}\text { Mycobacterium } \\
\text { tuberculosis }(\mathrm{H} 37 \mathrm{Rv} \\
\text { strain) }\end{array}$} & $6.25 \mu \mathrm{g} / \mathrm{mL}$ & \multirow{2}{*}[86]{} \\
\hline Tridax procumbens L. & & & $0.8 \mu \mathrm{g} / \mathrm{mL}$ & \\
\hline Triclisia gilletii & Methanol extract & $\begin{array}{l}\text { Mycobacterium } \\
\text { tuberculosis }\end{array}$ & 3.90 to $62.5 \mu \mathrm{g} / \mathrm{mL}$ & [87] \\
\hline Combretum hereroense & \multirow{3}{*}{$\begin{array}{c}\text { Hexane, } \\
\text { dichloromethane, } \\
\text { methanol, and acetone }\end{array}$} & \multirow{3}{*}{$\begin{array}{l}\text { M. smegmatis (ATCC } \\
\text { 1441), M. tuberculosis } \\
\text { (ATCC H37Rv) }\end{array}$} & $\begin{array}{c}1.6 \mathrm{mg} / \mathrm{mL} \text { and } 1.3 \\
\mathrm{mg} / \mathrm{mL}\end{array}$ & \multirow{3}{*}[88]{} \\
\hline Citrus lemon & & & $0.3 \mathrm{mg} / \mathrm{mL}$ & \\
\hline Apodytes dimidiata & & & $1.3 \mathrm{mg} / \mathrm{mL}$ & \\
\hline Cinnamomum verum & \multirow{2}{*}{$\begin{array}{l}\text { Aqueous \& methanolic } \\
\text { extracts }\end{array}$} & \multirow{2}{*}{$\begin{array}{l}\text { Mycobacterium } \\
\text { tuberculosis H37Rv }\end{array}$} & \multirow{2}{*}{$10 \mathrm{mcg} / \mathrm{mL}$} & \multirow{2}{*}{ [89] } \\
\hline Solanum surattense & & & & \\
\hline $\begin{array}{c}\text { Costus speciosus, Cymbopogon citratus, } \\
\text { and Tabernaemontana coronaria }\end{array}$ & Methanol extracts & $\begin{array}{l}\text { Mycobacterium } \\
\text { tuberculosis H37Rv }\end{array}$ & $100-200 \mu \mathrm{g} / \mathrm{mL}$ & [90] \\
\hline Croton tonkinensis & $\begin{array}{l}\text { Methylene chloride } \\
\text { extracts }\end{array}$ & $\begin{array}{c}\text { M. tuberculosis H37Ra, } \\
\text { H37Rv }\end{array}$ & 6.25 and $12.5 \mu \mathrm{g} / \mathrm{mL}$ & [91] \\
\hline $\begin{array}{l}\text { Melia azedarach L. and Lobelia } \\
\text { chinensis Lour. }\end{array}$ & $\begin{array}{l}\text { Methanol and n-hexane } \\
\text { extract }\end{array}$ & M. tuberculosis & $100 \mu \mathrm{g} / \mathrm{mL}$ & [92] \\
\hline
\end{tabular}

\section{Computational Analysis}

\subsection{Selection and Retrieval of Receptor Proteins}

The selection procedures of receptor proteins were purely based on the literature survey. To assess the multi-domain antimycobacterial activity of ligands, different types of receptor proteins (mycobacterial proteins) were taken into consideration that had different 3D structures and had different functions as well; specifically, they all must have had some critical functions required for the survival of target bacterial cell and its infection. The selected receptor proteins arabinosyltransferase C (PDB ID: 3PTY), protein kinase A (PDB ID: 4OW8), glutamine synthetase (PDB ID: 3ZXR), and proteasomal ATPase (PDB ID: 5KWA) all have some critical functions to perform the vital cellular functions. The arabinosyltransferase $\mathrm{C}$, belonging to enzyme class transferase, is a vital enzyme, playing a pivotal role in critical biological processes, and it participates in the biosynthesis of the essential part of the Mycobacterium cell wall [93]. Protein kinase A is recognized for its significant contribution in regulating the Mycobacterium cell shape and its mechanics. This protein gets exponentially upregulated during mycobacterial growth and infections [94]. Mycobacterial glutamine synthetase is known to increase the bacterium capacity to inhibit the host's phagosome-lysosome defense mechanism. It is also actively involved in cell wall biosynthesis and in converting glutamate, ammonia, and ATP to glutamine, phosphate, and ADP in bacterial cells [95]. At the same time, proteasomal ATPase from the bacterium is an essential virulent factor required for infection in humans [96]. Searching for a multi-domain mycobacterial inhibitory molecule, using all of the aforementioned receptor proteins, will aid in the discovery of novel inhibitory compounds with an overall inhibitory effect on the target bacterium. 
The crystal structures of receptor proteins were procured in the form of atomic coordinates from the Protein Data Bank (https:/ / www.rcsb.org; accessed on 25 October 2021), using specific PDB IDs of each protein. The unwanted water molecules, heteroatoms, and other ligand coordinates were removed from the protein structures to obtain a more suitable and stable conformation [97]. For the docking algorithm, along with the addition of polar hydrogens, Kollman charges were added to each protein molecule and saved in pdbqt format. The 3D structure of the receptor proteins is illustrated in Figure 1.

Arabinosyltransferase C

(PDB ID: 3PTY)

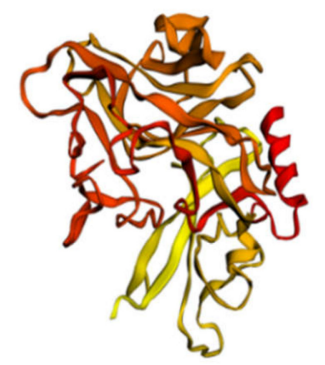

Proteasomal ATPase

(PDB ID: 5KWA)

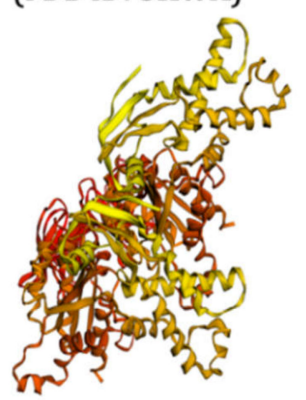

Glutamine Synthetase

(PDB ID: 3ZXR)

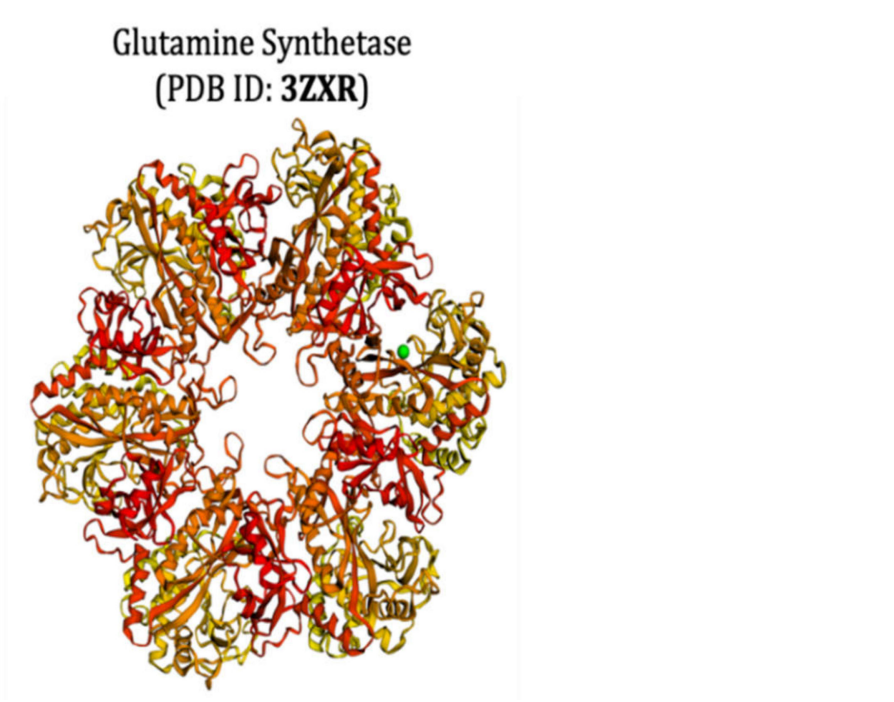

Protein kinase A

Figure 1. The 3D structure of the PDB-retrieved receptor proteins of M. tuberculosis.

\subsection{Selection and Retrieval of Ligand and Molecules}

Selections of the plant's secondary metabolites (test ligands) were based on the literature survey, inferring their biological activity for the well-being of human health. Alliin (S-allyl-L-cysteine sulfoxide), the most abundant sulfur compound in Allium sativum L., has been reported as a potent cardioprotective and neuroprotective agent having antidiabetic, anticholesteremic, and anticarcinogenic effects [98,99]. Aloe vera is a medicinal plant that exerts a hypoglycemic effect with no side effects [100]. Aloin, a major compound of $A$. vera latex, is a well-known laxative agent, generally existing as a mixture of two diastereoisomers, aloin A and aloin B, also referred to as barbaloin and isobarbaloin, respectively [101]. Phyllanthus emblica, commonly known as 'amLa' in India, has been used for treating various human ailments for centuries. The major bioactive compounds of P. emblica, including octyl$\beta$-D-glucopyranoside, have been well known for curing effects against varied diseases, such as fever, cough, piles, constipation, anorexia, hemorrhoids, skin diseases, asthma, biliousness, respiratory disorders, tumors, and cancer [102]. Phillips et al. [103] explained that the oleanolic acid isolated from Lantana hispida showed a potential inhibitory potential against $M$. tuberculosis H37RV (Table 2). Still, its inhibitory mechanism of action (MOA) is unknown; therefore, assessing its inhibitory activities against selected receptor proteins 
will give insight into its working MOA. (E)-phytol, a metabolite from Leucas volkensii, was also reported to have inhibitory activity against $M$. tuberculosis H37Rv (MIC: $2 \mu \mathrm{g} / \mathrm{mL}$ ) and is believed to be a better therapeutic agent for the treatment of TB [71,104,105].

The control ligands, i.e., isoniazid (ISN) and ethambutol (EMB), are used predominantly in the treatment of TB. However, they are known to cause severe side effects on patients and are failing in inhibitory termination of the Mycobacterium. Therefore, novel antimycobacterial molecules harboring less (or no) side effects are currently being searched for, and are highly desirable in current incidents of TB.

The native structures of all the ligand molecules were fetched from the PubChem database (https: / / pubchem.ncbi.nlm.nih.gov; accessed on 25 October 2021) using Chimera software and saved in pdbqt format. The 2D structures of all the ligands are presented in Figure 2.

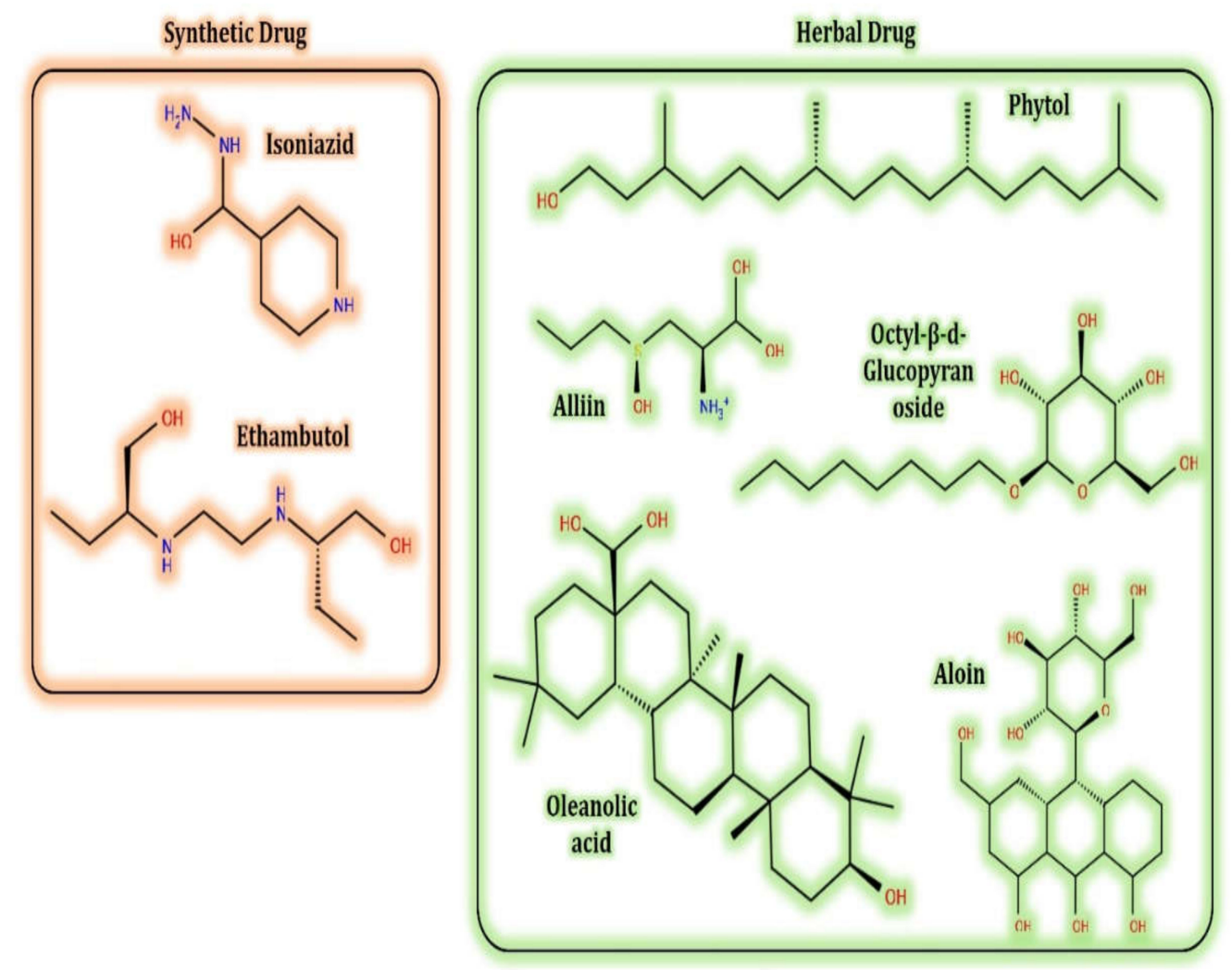

Figure 2. The 2D structure of the secondary metabolites from diverse plants.

\subsection{Docking Algorithm}

For the docking algorithm calculations, AutoDock 4.2 package (http://autodock. scripps.edu/resources/adt; accessed on 25 October 2021) was used. The ligand's native structures were blindly docked in the potential binding cavities of the receptor proteins. The AutoDock package executes the binding predictions of the ligands vs. receptor molecules based on the empirical force field and the Lamarckian genetic algorithm. The binding energy persuaded the binding affinity between the target sites and functional groups of ligand molecules utilizing various interactions viz. H-bonds, ionic interactions, hydrophobic interactions, and van der Waal's forces. With these understandings, the visual analysis of the docking algorithm executed between the selected ligands and receptor proteins is shown in Table 3. Chimera software was used to visualize the binding cascade of the ligand against the receptor protein complex. 
Table 3. Docking algorithm executed between the selected ligands and receptor proteins.

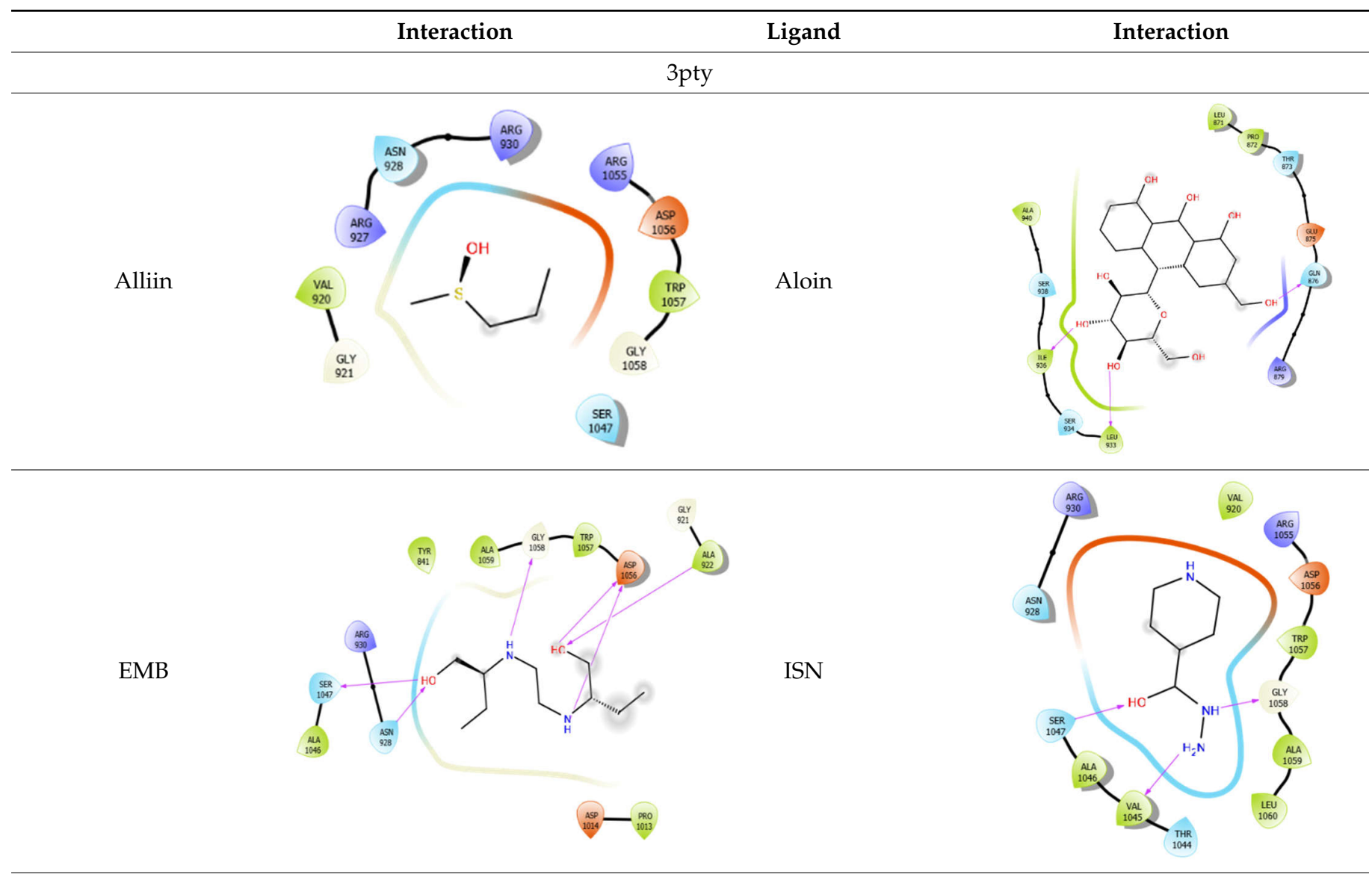

Octyl- $\beta-d-$

Glucopyranoside
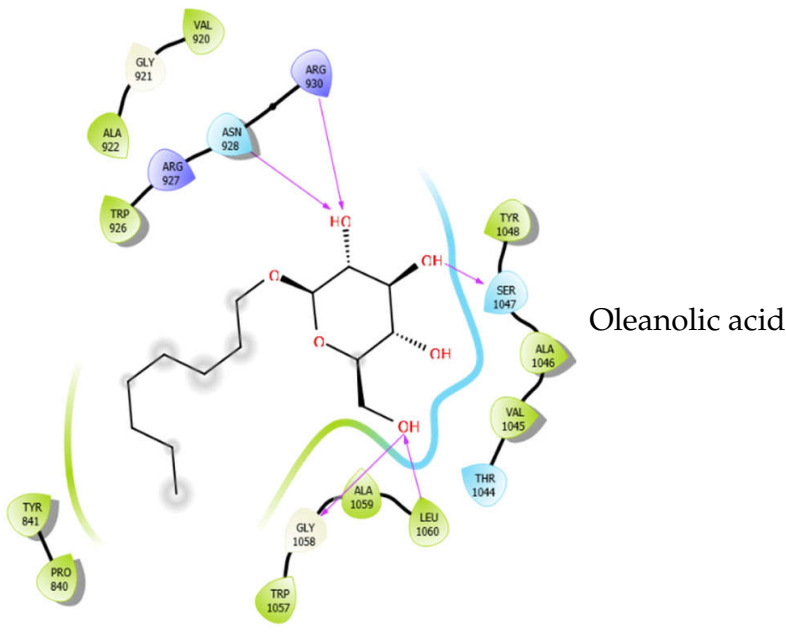

Phytol

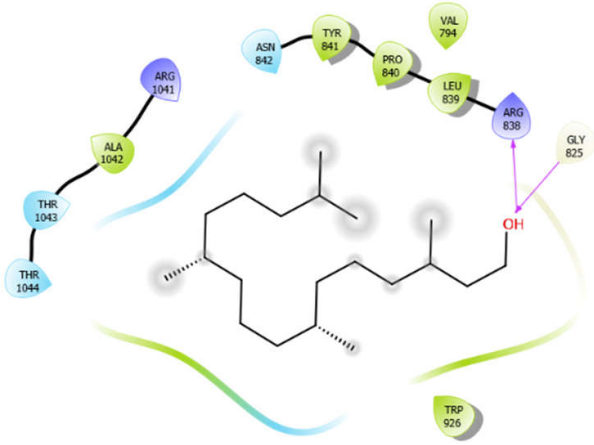


Table 3. Cont

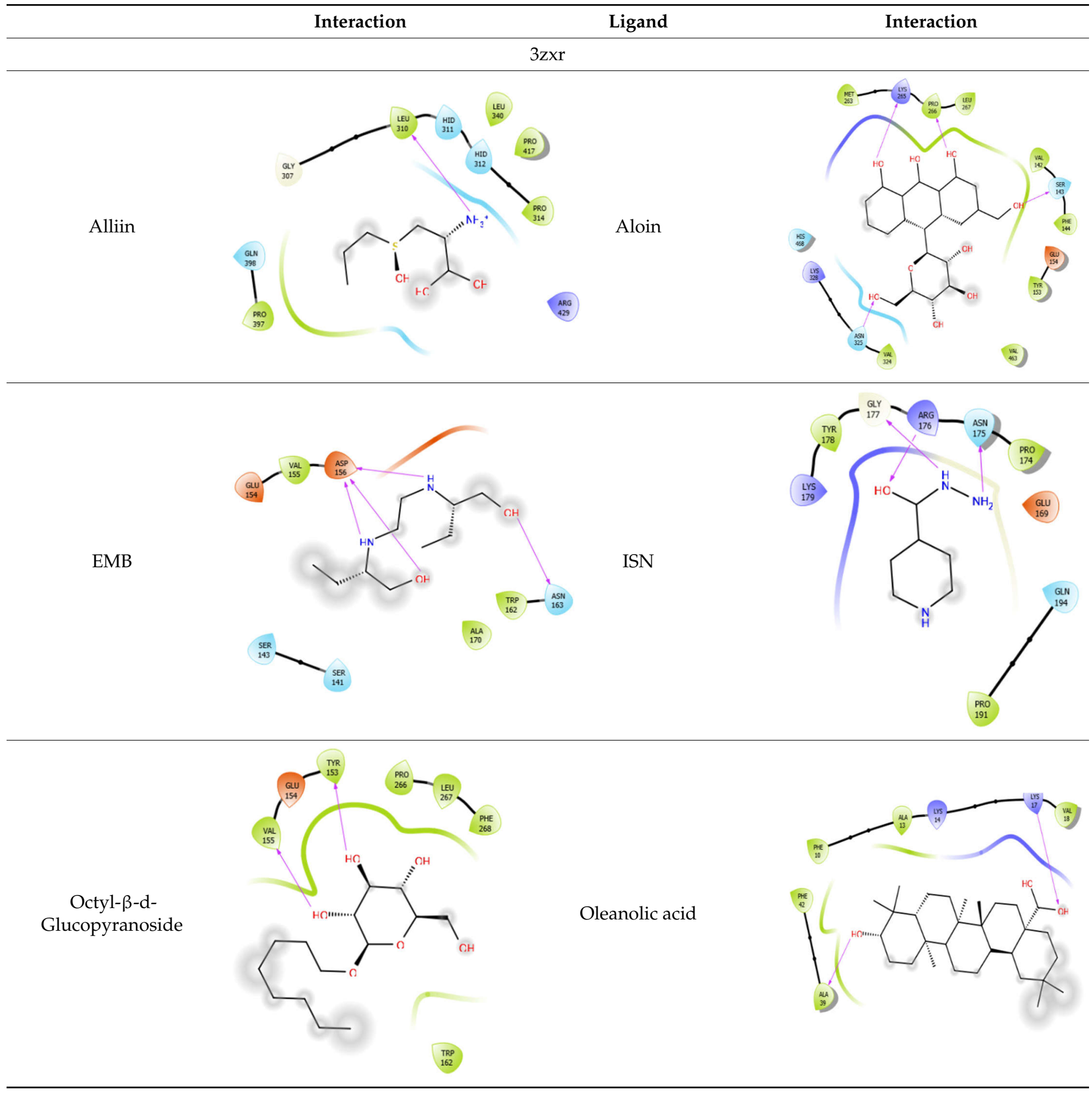


Table 3. Cont.

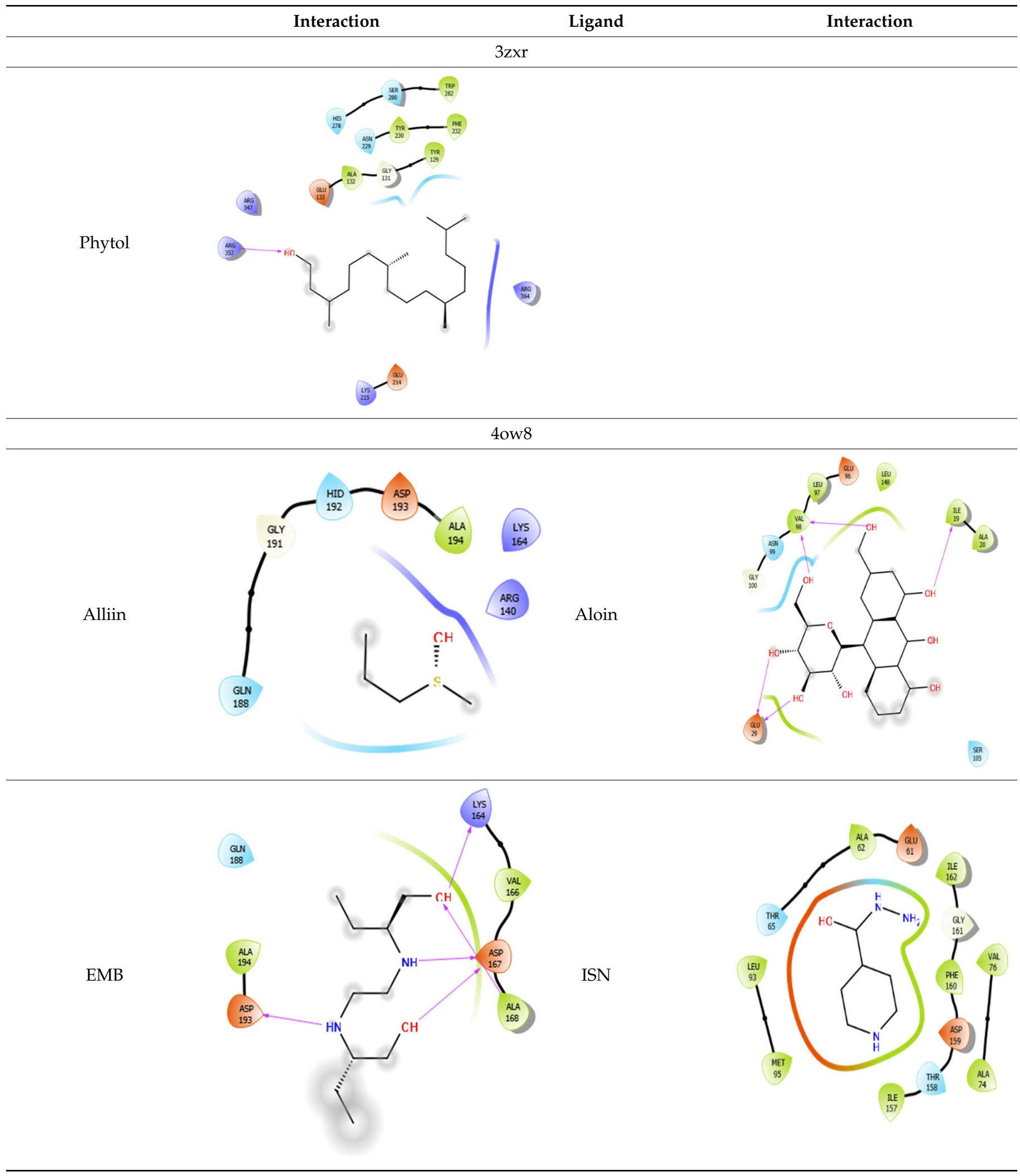


Table 3. Cont.

Interaction Interaction

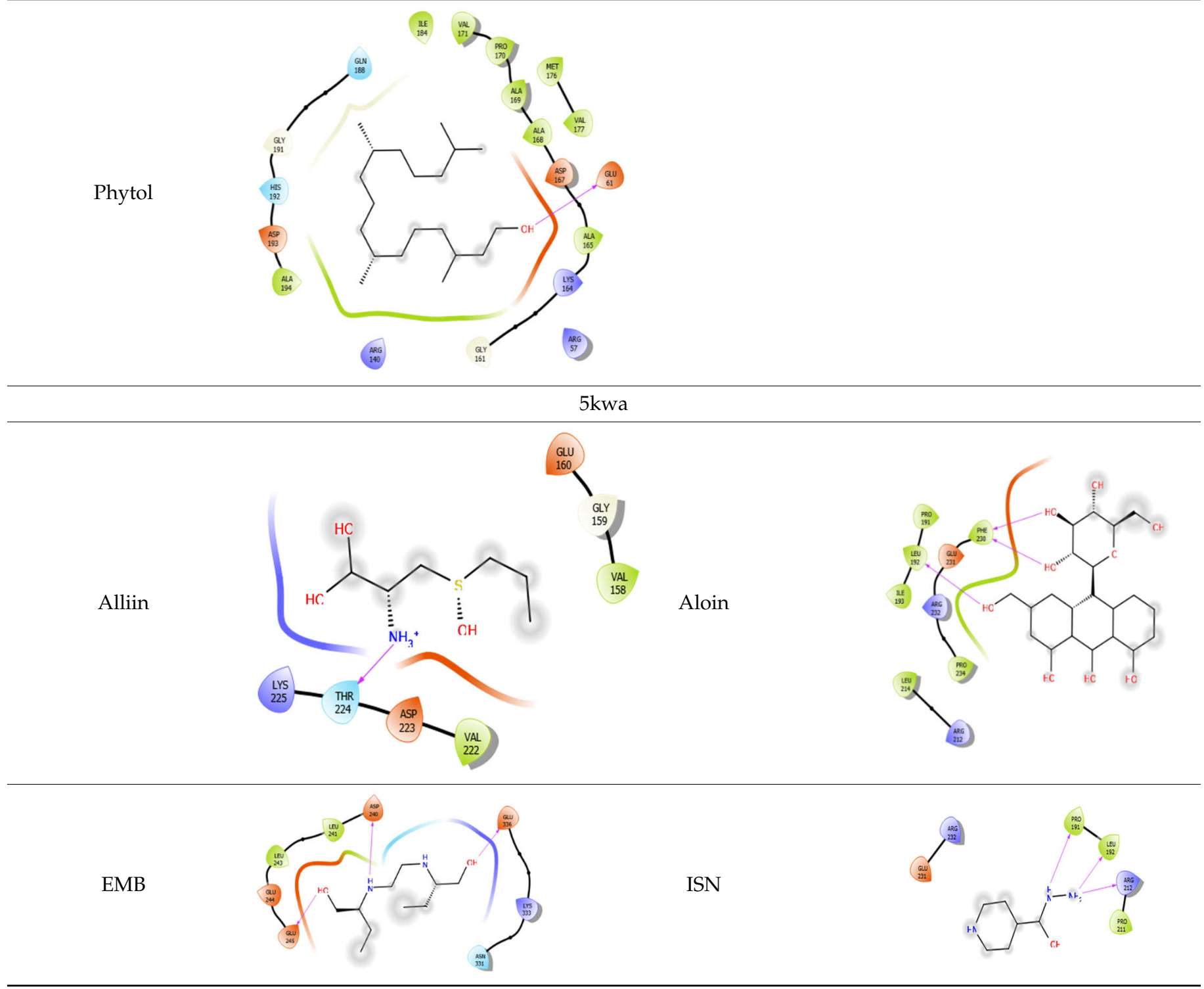


Table 3. Cont.

Interaction Interaction

The binding energy and inhibitory constant of ligands obtained against their interactions with receptor proteins are presented in Figure 3.

Binding Energy

$(\mathrm{kcal} / \mathrm{mol})$
Inhibition Constant

$(\mu \mathrm{M})$
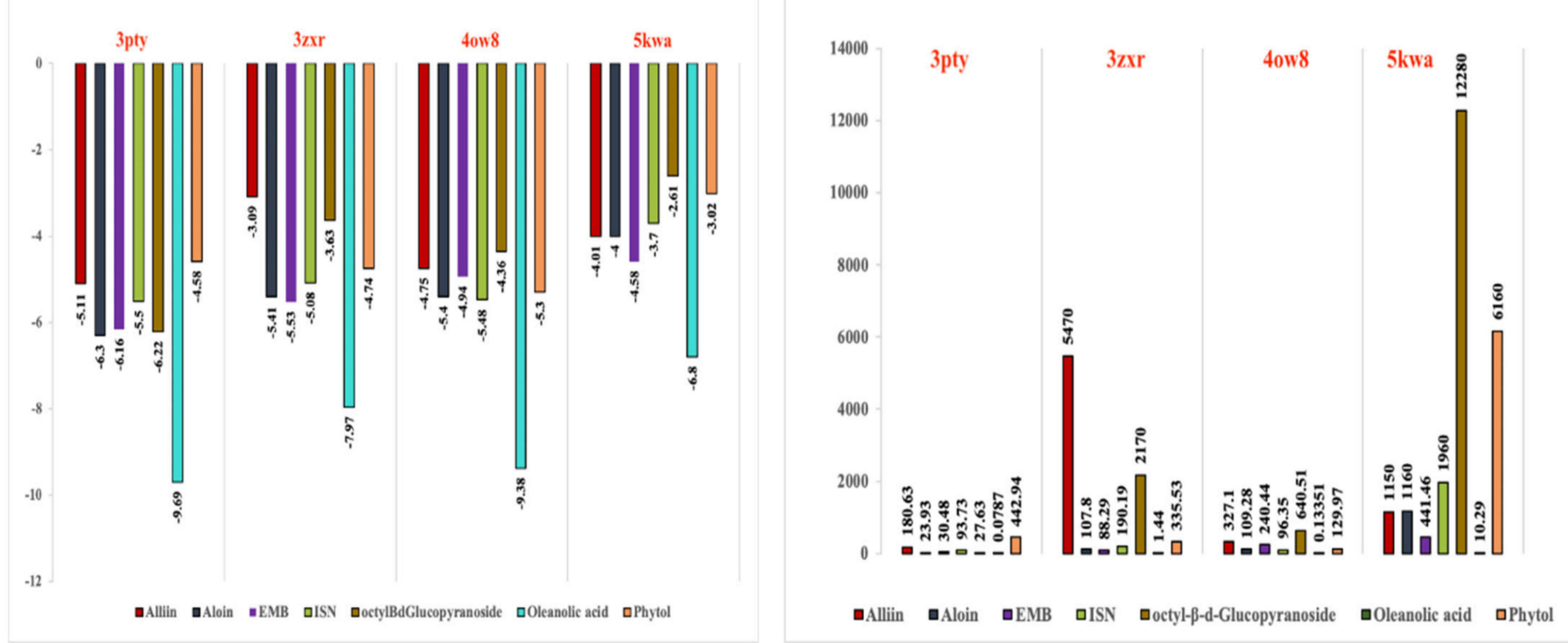

Figure 3. The binding energy and inhibitory constant of phytomolecules obtained after complexation with receptor proteins of M. tuberculosis (details also described in Supplementary Table S1).

Furthermore, more details regarding the interactive venture of ligands with active sites of the receptor protein can be seen in Table 4 . 
Table 4. The binding energy of interacted residues of receptors proteins of $M$. tuberculosis with diverse phytomolecules.

\begin{tabular}{|c|c|c|c|}
\hline Interactions & Bond Type & Resides and Their Legends & Binding Energy $(\mathrm{kcal} / \mathrm{mol})$ \\
\hline Alliin with 3pty & $\begin{array}{l}\text { Hydrogen bond } \\
\text { Hydrophobic bond } \\
\text { Polar bond } \\
\text { Charged bond }\end{array}$ & $\begin{array}{c}\text { GLY921, GLY1058 } \\
\text { VAL920, TRP1057 } \\
\text { ASN928, SER1047 } \\
\text { ARG927, ARG930, ARG1055, } \\
\text { ASP1056 }\end{array}$ & -5.11 \\
\hline Aloin with 3pty & $\begin{array}{l}\text { Hydrophobic bond } \\
\text { Polar bond } \\
\text { Charged bond }\end{array}$ & $\begin{array}{c}\text { LEU871, PRO872, LEU933, } \\
\text { ILE936, ALA940 } \\
\text { THR873, GUN876, SER934, } \\
\text { SER938 } \\
\text { GLU875, ARG879 }\end{array}$ & -6.3 \\
\hline EMB with 3pty & $\begin{array}{c}\text { Hydrogen bond } \\
\text { Hydrophobic bond } \\
\text { Polar bond } \\
\text { Charged bond }\end{array}$ & $\begin{array}{c}\text { GLY921, GLY1058 } \\
\text { TYR841, ALA922, PRO1013, } \\
\text { ALA1046, TRP1057, ALA1059, } \\
\text { ASN928, SER1047 } \\
\text { ARG930, ASP1014, ASP1056 }\end{array}$ & -6.16 \\
\hline ISN with 3pty & $\begin{array}{c}\text { Hydrogen bond } \\
\text { Hydrophobic bond } \\
\text { Polar bond } \\
\text { Charged bond }\end{array}$ & $\begin{array}{c}\text { GLY1058 } \\
\text { VAL920, VAL1045, ALA1046, } \\
\text { TRP1057, ALA1059, LEU1060 } \\
\text { ASN928, THR1044, SER1047 } \\
\text { ARG930, ARG1055, ASP1056 }\end{array}$ & -5.5 \\
\hline $\begin{array}{l}\text { Octyl- } \beta-d-\text {-Glucopyranoside } \\
\text { with 3pty }\end{array}$ & $\begin{array}{l}\text { Hydrogen bond } \\
\text { Hydrophobic bond } \\
\text { Polar bond } \\
\text { Charged bond }\end{array}$ & $\begin{array}{c}\text { GLY921, GLY1058 } \\
\text { PRO840, TYR841, VAL920, } \\
\text { ALA922, TRP926, VAL1045, } \\
\text { ALA1046, TYR1048, TRP1057, } \\
\text { ALA1059, LEU1060 } \\
\text { ASN928, THR1044, SER1047 } \\
\text { ARG927, ARG930 }\end{array}$ & -6.22 \\
\hline Oleanolic acid with 3pty & $\begin{array}{l}\text { Hydrophobic bond } \\
\text { Polar bond } \\
\text { Charged bond }\end{array}$ & $\begin{array}{l}\text { LEU871, PRO872, LEU933, } \\
\text { ILE936, PRO937, ALA940, } \\
\text { ILE965 } \\
\text { GLN876, SER938, THR939 } \\
\text { GLU875, ARG879 }\end{array}$ & -9.69 \\
\hline Phytol with 3pty & $\begin{array}{l}\text { Hydrogen bond } \\
\text { Hydrophobic bond } \\
\text { Polar bond } \\
\text { Charged bond }\end{array}$ & $\begin{array}{c}\text { GLY825 } \\
\text { ALA1042, TYR841, PRO840, } \\
\text { LEU839, TRP926 } \\
\text { THR1043, THR1044, ASN842 } \\
\text { ARG1041, ARG838 }\end{array}$ & -4.58 \\
\hline Alliin with 3zxr & $\begin{array}{l}\text { Hydrogen bond } \\
\text { Hydrophobic bond } \\
\text { Polar bond } \\
\text { Charged bond }\end{array}$ & $\begin{array}{c}\text { GLY307 } \\
\text { LEU310, PRO314, LEU340, } \\
\text { PRO417, PRO397 } \\
\text { HID311, HID312, GLN398 } \\
\text { ARG429 }\end{array}$ & -3.09 \\
\hline Aloin with 3zxr & $\begin{array}{l}\text { Hydrophobic bond } \\
\text { Polar bond } \\
\text { Charged bond }\end{array}$ & $\begin{array}{c}\text { MET263, PRO266, LEU267, } \\
\text { VAL142, PHE144, TYR153, } \\
\text { VAL463, VAL324 } \\
\text { SER143, HIS468, HIS468, } \\
\text { ASN325 } \\
\text { LYS265, LYS328, GLU154 }\end{array}$ & -5.41 \\
\hline EMB with $3 z x r$ & $\begin{array}{l}\text { Hydrophobic bond } \\
\text { Polar bond } \\
\text { Charged bond }\end{array}$ & $\begin{array}{c}\text { VAL155, TRP162, ALA170 } \\
\text { ASN163 } \\
\text { GLU154, ASP156 }\end{array}$ & -5.53 \\
\hline
\end{tabular}


Table 4. Cont.

\begin{tabular}{|c|c|c|c|}
\hline Interactions & Bond Type & Resides and Their Legends & Binding Energy (kcal/mol) \\
\hline ISN with $3 z x r$ & $\begin{array}{l}\text { Hydrogen bond } \\
\text { Hydrophobic bond } \\
\text { Polar bond } \\
\text { Charged bond }\end{array}$ & $\begin{array}{c}\text { GLY177 } \\
\text { TYR178, PRO174, PRO191 } \\
\text { ASN175, GLN194 } \\
\text { LSY179, ARG176, GLU169 }\end{array}$ & -5.08 \\
\hline $\begin{array}{l}\text { Octyl- } \beta \text {-d-glucopyranoside } \\
\text { with } 3 z x r\end{array}$ & $\begin{array}{c}\text { Hydrophobic bond } \\
\text { Polar bond } \\
\text { Charged bond }\end{array}$ & $\begin{array}{c}\text { VAL155, TYR153, PRO266, } \\
\text { LEU267, PHE268, TRP162 } \\
\text { HIS182 } \\
\text { GLU154 }\end{array}$ & -3.63 \\
\hline Oleanolic acid with 3zxr & $\begin{array}{l}\text { Hydrophobic bond } \\
\text { Charged bond }\end{array}$ & $\begin{array}{c}\text { PHE10, ALA13, VAL18, } \\
\text { PHE42, ALA39 } \\
\text { LYS14, LYS17 }\end{array}$ & -7.97 \\
\hline Phytol with 3zxr & $\begin{array}{c}\text { Hydrogen bond } \\
\text { Hydrophobic bond } \\
\text { Polar bond } \\
\text { Charged bond }\end{array}$ & $\begin{array}{c}\text { GLY131 } \\
\text { TRP282, TYR230, PHE232, } \\
\text { ALA132, TYR129 } \\
\text { HIS278, SER280, ASN229 } \\
\text { GLU133, ARG347, ARG352, } \\
\text { ARG364, GLU214, LYS215 }\end{array}$ & -4.74 \\
\hline Alliin with 4ow8 & $\begin{array}{l}\text { Hydrogen bond } \\
\text { Hydrophobic bond } \\
\text { Polar bond } \\
\text { Charged bond }\end{array}$ & $\begin{array}{c}\text { GLY191 } \\
\text { ALA194 } \\
\text { GLN188, HID192 } \\
\text { ASP193, LYS164, ARG140 }\end{array}$ & -4.75 \\
\hline Aloin with 4ow8 & $\begin{array}{c}\text { Hydrogen bond } \\
\text { Hydrophobic bond } \\
\text { Polar bond } \\
\text { Charged bond }\end{array}$ & $\begin{array}{c}\text { GLY100 } \\
\text { VAL98, LEU97, LEU148, } \\
\text { ILE19, ALA20 } \\
\text { ASN99 } \\
\text { GLU96, GLU29 }\end{array}$ & -5.4 \\
\hline EMB with 4 ow 8 & $\begin{array}{c}\text { Hydrophobic bond } \\
\text { Polar bond } \\
\text { Charged bond }\end{array}$ & $\begin{array}{c}\text { ALA194, VAL166, ALA168 } \\
\text { GLN188 } \\
\text { ASP193, LYS164, ASP167 }\end{array}$ & -4.94 \\
\hline ISN with 4ow8 & $\begin{array}{l}\text { Hydrogen bond } \\
\text { Hydrophobic bond } \\
\text { Polar bond } \\
\text { Charged bond }\end{array}$ & $\begin{array}{c}\text { GLY161 } \\
\text { MET95, LEU93, ALA62, } \\
\text { ILE162, PHE160, ILE157, } \\
\text { VAL76, ALA74 } \\
\text { THR65, THR158 } \\
\text { GLU61, ASP159 }\end{array}$ & -5.48 \\
\hline $\begin{array}{l}\text { Octyl- } \beta \text {-d-glucopyranoside } \\
\text { with } 4 \text { ow } 8\end{array}$ & $\begin{array}{l}\text { Hydrogen bond } \\
\text { Hydrophobic bond } \\
\text { Polar bond } \\
\text { Charged bond }\end{array}$ & $\begin{array}{c}\text { GLY191 } \\
\text { VAL166, ALA168, ALA169, } \\
\text { PRO170, VAL171, ALA194, } \\
\text { MET176, VAL177, MET178 } \\
\text { HIS192, GLN188 } \\
\text { LYS164, ASP167 }\end{array}$ & -4.36 \\
\hline Oleanolic acid with 4ow8 & $\begin{array}{c}\text { Hydrophobic bond } \\
\text { Polar bond }\end{array}$ & $\begin{array}{c}\text { PRO216, PHE217, ALA218, } \\
\text { PRO238, LEU237, PRO236, } \\
\text { PRO235, TYR208 } \\
\text { SER212 } \\
\text { LYS214, ARG215, LYS228 }\end{array}$ & -9.38 \\
\hline Phytol with 4ow8 & $\begin{array}{l}\text { Hydrogen bond } \\
\text { Hydrophobic bond } \\
\text { Polar bond } \\
\text { Charged bond }\end{array}$ & $\begin{array}{c}\text { GLY191, GLY161 } \\
\text { ALA194, ILE184, VAL171, } \\
\text { PRO170, ALA169, ALA168, } \\
\text { ALA165, MET176, VAL177 } \\
\text { GLN188, HIS192 } \\
\text { ASP193, ASP167, LYS164, } \\
\text { GLU61, ARG57, ARG140 }\end{array}$ & -5.3 \\
\hline
\end{tabular}


Table 4. Cont.

\begin{tabular}{|c|c|c|c|}
\hline Interactions & Bond Type & Resides and Their Legends & Binding Energy (kcal/mol) \\
\hline Alliin with 5kwa & $\begin{array}{l}\text { Hydrogen bond } \\
\text { Hydrophobic bond } \\
\text { Polar bond } \\
\text { Charged bond }\end{array}$ & $\begin{array}{c}\text { GLY159 } \\
\text { VAL158, VAL222 } \\
\text { THR224 } \\
\text { GLU160, LYS225, ASP223 }\end{array}$ & -4.01 \\
\hline Aloin with 5kwa & $\begin{array}{l}\text { Hydrophobic bond } \\
\text { Charged bond }\end{array}$ & $\begin{array}{l}\text { PRO191, LEU192, ILE193, } \\
\text { PHE230, PRO234, LEU214 } \\
\text { GLU231, ARG232, ARG212 }\end{array}$ & -4.0 \\
\hline EMB with 5kwa & $\begin{array}{l}\text { Hydrophobic bond } \\
\text { Polar bond } \\
\text { Charged bond }\end{array}$ & $\begin{array}{c}\text { LEU241, LEU243 } \\
\text { ASN331 } \\
\text { ASP240, GLU244, GLU245, } \\
\text { GLU336, LYS333 }\end{array}$ & -4.58 \\
\hline ISN with $5 \mathrm{kwa}$ & $\begin{array}{l}\text { Hydrophobic bond } \\
\text { Charged bond }\end{array}$ & $\begin{array}{l}\text { PRO191, LEU192, PRO211 } \\
\text { ARG232, GLU231, ARG212 }\end{array}$ & -3.7 \\
\hline $\begin{array}{l}\text { Octyl- } \beta \text {-d-glucopyranoside } \\
\text { with } 5 \mathrm{kwa}\end{array}$ & $\begin{array}{l}\text { Hydrogen bond } \\
\text { Hydrophobic bond } \\
\text { Polar bond } \\
\text { Charged bond }\end{array}$ & $\begin{array}{c}\text { GLY296, GLY298, GLY516 } \\
\text { CYS297, LEU301, ALA517 } \\
\text { THR300, ASN416 } \\
\text { LYS299, ASP371, GLU372 }\end{array}$ & -2.61 \\
\hline Oleanolic acid with $5 \mathrm{kwa}$ & $\begin{array}{l}\text { Hydrophobic bond } \\
\text { Polar bond } \\
\text { Charged bond }\end{array}$ & $\begin{array}{l}\text { PRO285, LEU283, PRO271 } \\
\text { SER282 } \\
\text { ARG284, ARG279, GLU407, } \\
\text { LYS275 }\end{array}$ & -6.8 \\
\hline Phytol with 5kwa & $\begin{array}{l}\text { Polar bond } \\
\text { Charged bond }\end{array}$ & $\begin{array}{c}\text { LEU561, PRO562, PRO567, } \\
\text { TRP570, ILE599 } \\
\text { ASN563, THR565, ASN566, } \\
\text { THR601 } \\
\text { ASP560, ARG597, ASP600 }\end{array}$ & -3.02 \\
\hline
\end{tabular}

Among all of the test ligands analyzed, oleanolic acid performed best in multi-regimen inhibition of Mycobacterium. It showed efficient binding, with all four receptor proteins having the most negative binding energies among all ligand-receptor interactions. Energetically, oleanolic acid vs. arabinosyltransferase $\mathrm{C}$ was the most favored interaction, having a binding energy of $-9.69 \mathrm{kcal} / \mathrm{mol}$. Other test ligands viz. alliin, aloin, octyl- $\beta$-Dglucopyranoside, and (E)-phytol showed similar affinity towards the receptor proteins as the control ligands, ISN and EMB. Despite exhibiting antimycobacterial activity, (E)-phytol did not perform as expected against the selected receptor proteins. However, all of the test ligands presented efficient multi-domain inhibitory functions against the Mtb.

The inhibition constant value $(\mathrm{Ki})$ of all ligand-receptor interactions indicates the inhibitory potential of the ligands against the selected receptor protein. The Ki value represents the ligand concentration required to inhibit the activity of half of the amount of receptor proteins. It is inversely proportional to the binding affinity of the ligands. Oleanolic acid showed the lowest possible $\mathrm{Ki}$ against all of the receptor proteins among the inhibitory potential of the ligands. In the oleanolic acid vs. arabinosyltransferase $C$ interaction complex, the lowest Ki value, i.e., $0.0787 \mu \mathrm{M}$, was observed. All other test ligands, except octyl- $\beta$-D-glucopyranoside, performed similarly with the control ligands.

\subsection{Toxicity Assay of Test Ligands}

The cytotoxicity of test ligands was assessed via the in silico web facility ProTox-II (http:/ / tox.charite.de/protox_II; accessed on 25 October 2021) and found to be ineffective against humans. 


\section{Bioinformatics Opportunities for Medicinal Plant Studies}

Plants have been used as therapeutic regimens since immemorial periods, and various commercially significant medicines are derived from plants. However, traditional methods used to explore plant-based regimens are timely and are highly expensive. Moreover, such extensive works have faced several problems in keeping up with the hasty advancement of high throughput technologies. In this era of high volume, high-throughput data production in life sciences-bioinformatics plays an essential role in overcoming the above-mentioned problems, with limited time and expenditure in drug design and discovery [106,107].

Nowadays, bioinformatics plays a crucial role in exploring the role of medicinal plants against various diseases, diabetes, cancer, and tuberculosis. With ever-increasing genomic and proteomic studies, it is essential to decipher the data competently. Bioinformatics plays a crucial role in exploring new genetic factors, driving the identification of several new genes and proteins. In addition, its tools have aided in explaining significant relationships between several molecular factors [108]. Thus, bioinformatic approaches, such as molecular docking, RMSD value, etc., help in the screening of plant metabolites, to develop drugs that target virulent factors associated with molecular pathways.

\section{Concluding Remarks and Future Directions}

TB is a severe disease; its treatment started 73 years after the discovery of streptomycin and other drugs. TB is also one of the world's top- ten infectious diseases. The emergence of MDR/XDR/TDR-Mtb strains has worsened the situation, causing a severe threat to human health. The present research claims that chemotherapy using synthetic anti-tuberculosis drugs is not very efficient at killing the dormant and intracellular forms of Mtb. Researchers are focusing on secondary metabolites in plants due to their therapeutic potential. However, there is still a need to resolve some concerns, for better application of secondary metabolites and to effectively manage human diseases (including tuberculosis). Major concerns include (a) lower animal experimentation facilities used for investigating the in vivo effects of phytomolecules; (b) lower solubility of the natural products; and (c) unavailability of sufficient amounts of pure compounds. Considering the above, there is a need to identify more anti-tuberculosis phytomolecules that have selective neutralizing activities against specific target proteins, using computational and bioinformatics approaches (Figure 3). The application of improved extraction procedures, high throughput techniques for precise determination of bioactive molecules, and structural elucidation of novel chemical molecules expressing potential effectiveness against different clinical isolates and isolates with MDR/XDR/TDR characteristics, is highly imperative. The extensive investigation of in vitro mechanisms of action of molecules showing better outcomes under in silico studies could help design molecules and analogs with minimal side effects. Information pertaining to important proteins participating in the survival of mycobacteria in the host system and a complete understanding of biosynthetic pathways and genetic regulation might facilitate the development of natural products with considerable inhibitory action against Mycobacterium tuberculosis and other species. In addition, the in vitro cytotoxicity of plant metabolites should be considered while searching for new candidate molecules with potential anti-tuberculosis effects. Such studies would not only solve the disease burden of TB by identifying the novel structures of variant compounds against resistant and nonresistant Mtb strains, but would also facilitate raising the importance of the therapeutic properties of medicinal plants in modern medicine.

Supplementary Materials: The following are available online at https: / www.mdpi.com/article/ 10.3390/antiox10121990/s1, Table S1: The binding energy of interacted residues of receptors proteins of M. tuberculosis with diverse Phytomolecules.

Author Contributions: M.K., A.K. and S.K.S. designed the study; M.K., A.K., S.K.S., P.P.S. wrote the manuscript; M.K. acquired funding; M.K., A.K., S.-M.C. supervised the study; V.K.S., A.K.S., L.S., A.C.R., M.S.K., A.K.J., S.-M.C. provided valuable feedback. All authors have read and agreed to the published version of the manuscript. 
Funding: M.K. would like to thank Dongguk University 2020 for funding.

Institutional Review Board Statement: Not applicable.

Informed Consent Statement: Not applicable.

Data Availability Statement: Data is available within the article.

Acknowledgments: The authors are thankful to Dongguk University and the Agriculture Research Organization for providing lab facilities.

Conflicts of Interest: The authors declare no conflict of interest.

$\begin{array}{ll}\text { Abbreviations } \\ \text { MDR } & \text { multi-drug resistance } \\ \text { SMs } & \text { secondary metabolites } \\ \text { TB } & \text { tuberculosis } \\ \text { XDR } & \text { extensively drug resistance } \\ \text { TDR } & \text { total drug resistance } \\ \text { Mtb } & \text { Mycobacterium tuberculosis } \\ \text { DPPH } & \text { 2,2-diphenyl-1-picryl-hydrazyl-hydrate } \\ \text { ABTS } & \text { 2,2'-azino-bis(3-ethylbenzothiazoline-6-sulfonic acid } \\ \text { ISN } & \text { isoniazid } \\ \text { EMB } & \text { ethambutol } \\ \text { INH } & \text { isonicotinic acid hydrazide } \\ \text { ATCC } & \text { American Type Culture Collection } \\ \text { PDB } & \text { Protein Data Bank } \\ \text { MOA } & \text { mechanism of action } \\ \text { RMSD } & \text { root mean square deviation }\end{array}$

\section{References}

1. Chin, Y.-W.; Balunas, M.J.; Chai, H.B.; Kinghorn, A.D. Drug discovery from natural sources. AAPS J. 2006, 8, E239-E253. [CrossRef] [PubMed]

2. Newman, D.J.; Cragg, G.M. Natural Products as Sources of New Drugs over the 30 Years from 1981 to 2010. J. Nat. Prod. 2012, 75 , 311-335. [CrossRef] [PubMed]

3. Boucher, H.W.; Ambrose, P.G.; Chambers, H.F.; Ebright, R.H.; Jezek, A.; Murray, B.E.; Newland, J.G.; Ostrowsky, B.; Rex, J.H.; Infectious Diseases Society of America. White Paper: Developing Antimicrobial Drugs for Resistant Pathogens, Narrow-Spectrum Indications, and Unmet Needs. J. Infect. Dis. 2017, 216, 228-236. [CrossRef] [PubMed]

4. Anand, U.; Jacobo-Herrera, N.J.; Altemimi, A.B.; Lakhssassi, N. A Comprehensive Review on Medicinal Plants as Antimicrobial Therapeutics: Potential Avenues of Biocompatible Drug Discovery. Metabolites 2019, 9, 258. [CrossRef]

5. WHO. Tuberculosis. Available online: https://www.who.int/news-room/fact-sheets/detail/tuberculosis (accessed on 26 October 2021).

6. Temesgen, E.; Belete, Y.; Haile, K.; Ali, S. Prevalence of active tuberculosis and associated factors among people with chronic psychotic disorders at St. Amanuel Mental Specialized Hospital and Gergesenon Mental Rehabilitation center, Addis Ababa, Ethiopia. BMC Infect. Dis. 2021, 21, 1100. [CrossRef]

7. Sharma, D.; Yadav, J.P. An Overview of Phytotherapeutic Approaches for the Treatment of Tuberculosis. Mini Rev. Med. Chem. 2016, 17, 167-183. [CrossRef]

8. Bunalema, L.; Fotso, G.W.; Waako, P.; Tabuti, J.; Yeboah, S.O. Potential of Zanthoxylum leprieurii as a source of active compounds against drug resistant Mycobacterium tuberculosis. BMC Complement. Altern. Med. 2017, 17, 89. [CrossRef]

9. Jamkhande, P.G.; Pathan, S.K. In silico PASS analysis and determination of antimycobacterial, antifungal, and antioxidant efficacies of maslinic acid in an extract rich in pentacyclic triterpenoids. Int. J. Mycobacteriol. 2016, 5, 417-425. [CrossRef]

10. Elisha, I.L.; Botha, F.S.; McGaw, L.J.; Eloff, J.N. The antibacterial activity of extracts of nine plant species with good activity against Escherichia coli against five other bacteria and cytotoxicity of extracts. BMC Complement. Altern. Med. 2017, 17, 133. [CrossRef]

11. Robinson, M.M.; Zhang, X. The World Medicines Situation 2011, Traditional Medicines: Global Situation, Issues and Challenges; World Health Organization: Geneva, Switzerland, 2011; pp. 1-2.

12. Teoh, E.S. Secondary Metabolites of Plants. In Medicinal Orchids of Asia; Springer: Cham, Switzerland, 2016 ; pp. 59-73.

13. Gorlenko, C.L.; Kiselev, H.Y.; Budanova, E.V.; Zamyatnin, J.A.A.; Ikryannikova, L.N. Plant Secondary Metabolites in the Battle of Drugs and Drug-Resistant Bacteria: New Heroes or Worse Clones of Antibiotics? Antibiototics 2020, 9, 170. [CrossRef]

14. Dias, D.A.; Urban, S.; Roessner, U. A Historical Overview of Natural Products in Drug Discovery. Metabolites 2012, 2, 303-336. [CrossRef] 
15. Othman, L.; Sleiman, A.; Abdel-Massih, R.M. Antimicrobial Activity of Polyphenols and Alkaloids in Middle Eastern Plants. Front. Microbiol. 2019, 10, 911. [CrossRef]

16. Lü, J.-M.; Lin, P.H.; Yao, Q.; Chen, C. Chemical and molecular mechanisms of antioxidants: Experimental approaches and model systems. J. Cell. Mol. Med. 2010, 14, 840-860. [CrossRef]

17. Venkatachalam, R.; Kalimuthu, K.; Chinnadurai, V.; Saravanan, M.; Radhakrishnan, R.; Shanmuganathan, R.; Pugazhendhi, A. Various solvent effects on phytochemical constituent profiles, analysis of antioxidant and antidiabetic activities of Hopea parviflora. Process. Biochem. 2020, 89, 227-232. [CrossRef]

18. Magalhães, L.M.; Barreiros, L.; Reis, S.; Segundo, M.A. Kinetic matching approach applied to ABTS assay for high-throughput determination of total antioxidant capacity of food products. J. Food Compos. Anal. 2014, 33, 187-194. [CrossRef]

19. Pavithra, G.M.; Siddiqua, S.; Naik, A.S.; TR, P.K.; Vinayaka, K.S. Antioxidant and antimicrobial activity of flowers of Wendlandia thyrsoidea, Olea dioica, Lagerstroemia speciosa and Bombax malabaricum. J. Appl. Pharmac. Sci. 2013, 3, 114. [CrossRef]

20. Yahia, Y.; Benabderrahim, M.A.; Tlili, N.; Bagues, M.; Nagaz, K. Bioactive compounds, antioxidant and antimicrobial activities of extracts from different plant parts of two Ziziphus Mill. species. PLoS ONE 2020, 15, e0232599. [CrossRef]

21. Wagay, N.A.; Khan, N.A.; Rothe, S.P. Profiling of secondary metabolites and antimicrobial activity of Crateva religiosa G. Forst. bark-A rare medicinal plant of Maharashtra India. Int. J. Biosci. 2017, 10, 343-354. [CrossRef]

22. Köksal, E.; Tohma, H.; Kılıç, Ö.; Alan, Y.; Aras, A.; Gülçin, I.; Bursal, E. Assessment of Antimicrobial and Antioxidant Activities of Nepeta trachonitica: Analysis of Its Phenolic Compounds Using HPLC-MS/MS. Sci. Pharm. 2017, 85, 24. [CrossRef]

23. Yu, M.; Gouvinhas, I.; Rocha, J.; Barros, A.I.R.N.A. Phytochemical and antioxidant analysis of medicinal and food plants towards bioactive food and pharmaceutical resources. Sci. Rep. 2021, 11, 10041. [CrossRef]

24. Elvin-Lewis, M.; Lewis, W.H. New Concepts and Medical and Dental Ethnobotany; Dioscorides Press: Portland, OR, USA, 1995; pp. 303-310.

25. Jordan, M.A.; Wilson, L. Microtubules as a target for anticancer drugs. Nat. Rev. Cancer 2004, 4, 253-265. [CrossRef] [PubMed]

26. Reyburn, H.; Mtove, G.; Hendriksen, I.; Von Seidlein, L. Oral quinine for the treatment of uncomplicated malaria. BMJ 2009, 339, b2066. [CrossRef] [PubMed]

27. Rodney, C.; Toni, M.; Kutchan, N.; Lewis, G. Biochemistry and Molecular Biology of Plants. In Natural Products; Buchanan, B., Gruissem, W., Jones, R., Eds.; Wiley: Rockville, MD, USA, 2000; pp. 1253-1348.

28. Rauha, J.-P.; Remes, S.; Heinonen, M.; Hopia, A.; Kähkönen, M.; Kujala, T.; Pihlaja, K.; Vuorela, H.; Vuorela, P. Antimicrobial effects of Finnish plant extracts containing flavonoids and other phenolic compounds. Int. J. Food Microbiol. 2000, 56, 3-12. [CrossRef]

29. Perez, D.R.; Lim, W.; Seiler, J.P.; Yi, G.; Peiris, M.; Shortridge, K.F.; Webster, R.G. Role of Quail in the Interspecies Transmission of H9 Influenza A Viruses: Molecular Changes on HA That Correspond to Adaptation from Ducks to Chickens. J. Virol. 2003, 77, 3148-3156. [CrossRef] [PubMed]

30. Santos, A.; De Campos, R.O.; Miguel, O.G.; Filho, V.C.; Siani, A.C.; Yunes, R.A.; Calixto, J.B. Antinociceptive properties of extracts of new species of plants of the genus Phyllanthus (Euphorbiaceae). J. Ethnopharmacol. 2000, 72, 229-238. [CrossRef]

31. Nichenametla, S.; Taruscio, T.G.; Barney, D.L.; Exon, J.H. A Review of the Effects and Mechanisms of Polyphenolics in Cancer. Crit. Rev. Food Sci. Nutr. 2006, 46, 161-183. [CrossRef]

32. Prats, E.; Galindo, J.C.; Bazzalo, M.E.; León, A.; Macías, F.A.; Rubiales, D.; Jorrín, J.V. Antifungal Activity of a New Phenolic Compound from Capitulum of a Head Rot-resistant Sunflower Genotype. J. Chem. Ecol. 2007, 33, 2245-2253. [CrossRef]

33. Okunade, A.L.; Hufford, C.D.; Clark, A.M.; Lentz, D. Antimicrobial properties of the constituents of Piper aduncum. Phytotherapy Research: An International. J. Devoted Med. Sci. Res. Plants Plant Prod. 1997, 11, 142-144.

34. Kato, T.; Tsuda, H.; Ishitani, Y.; Takemura, Y.; Suzuki, Y. 6-Acetyl-8-hydroxy-2,2-dimethylchromene, an Antioxidant in Sunflower Seeds; Its Isolation and Synthesis and Antioxidant Activity of Its Derivatives. Heterocycles 1997, 44, 139. [CrossRef]

35. Olsson, M.E.; Gustavsson, K.E.; Andersson, S.; Nilsson, A.; Duan, R.D. Inhibition of Cancer Cell Proliferation in Vitro by Fruit and Berry Extracts and Correlations with Antioxidant Levels. J. Agric. Food Chem. 2004, 52, 7264-7271. [CrossRef]

36. Swargiary, A.; Verma, A.K.; Singh, S.; Roy, M.K.; Daimari, M. Antioxidant and Antiproliferative Activity of Selected Medicinal Plants of Lower Assam, India: An In Vitro and In Silico Study. Anti-Cancer Agents Med. Chem. 2021, 21, 267-277. [CrossRef]

37. Meghashree, K.S.; Latha, K.P.; Vagdevi, H.M. Antioxidant and antitubercular activities of leaf extracts of Canthium dicoccum (Gaertn.) and Amischophacelus axillaris(L.). Indian J. Nat. Prod. Resour. 2021, 11, 244-249.

38. Hussain, K.; Ismail, Z.; Sadikun, A.; Ibrahim, P. Antioxidant, anti-TB activities, phenolic and amide contents of standardised extracts of Piper sarmentosum Roxb. Nat. Prod. Res. 2009, 23, 238-249. [CrossRef]

39. Trevizan, L.N.F.; do Nascimento, K.F.; Santos, J.A.; Kassuya, C.A.L.; Cardoso, C.A.L.; do Carmo Vieira, M.; Moreira, F.M.F.; Croda, J.; Formagio, A.S.N. Anti-inflammatory, antioxidant and anti-Mycobacterium tuber-culosis activity of viridiflorol: The major constituent of Allophylus edulis (A. St.-Hil., A. Juss. \& Cambess.). Radlk. J. Ethnopharmacol. 2016, 192, 510-515.

40. Khlifi, D.; Hamdi, M.; El Hayouni, A.; Cazaux, S.; Souchard, J.P.; Couderc, F.; Bouajila, J. Global Chemical Composition and Antioxidant and Anti-Tuberculosis Activities of Various Extracts of Globularia alypum L. (Globulariaceae) Leaves. Molecules 2011, 16, 10592-10603. [CrossRef]

41. Tawde, K.; Gacche, R.; Pund, M. Evaluation of selected Indian traditional folk medicinal plants against Mycobacterium tuberculosis with antioxidant and cytotoxicity study. Asian Pac. J. Trop. Dis. 2012, 2, S685-S691. [CrossRef] 
42. Janmanchi, H.; Raju, A.; Degani, M.; Ray, M.; Rajan, M. Antituberculosis, antibacterial and antioxidant activities of Aegiceras corniculatum, a mangrove plant and effect of various extraction processes on its phytoconstituents and bioactivity. S. Afr. J. Bot. 2017, 113, 421-427. [CrossRef]

43. Qamar, M.T.U.; Maryam, A.; Muneer, I.; Xing, F.; Ashfaq, U.A.; Khan, F.A.; Anwar, F.; Geesi, M.H.; Khalid, R.R.; Rauf, S.A.; et al. Computational screening of medicinal plant phytochemicals to discover potent pan-serotype inhibitors against dengue virus. Sci. Rep. 2019, 9, 1433. [CrossRef]

44. Kitchen, D.B.; Decornez, H.; Furr, J.R.; Bajorath, J. Docking and scoring in virtual screening for drug discovery: Methods and applications. Nat. Rev. Drug Discov. 2004, 3, 935-949. [CrossRef]

45. Ghosh, A.K.; Gemma, S. Structure-Based Design of Drugs and Other Bioactive Molecules: Tools and Strategies; John Wiley \& Sons: New York, NY, USA, 2014.

46. Hopkins, A.L. Network pharmacology: The next paradigm in drug discovery. Nat. Chem. Biol. 2008, 4, 682-690. [CrossRef]

47. Sandeep, G.; Nagasree, K.P.; Hanisha, M.; Kumar, M.M.K. AUDocker LE: A GUI for virtual screening with AUTODOCK Vina. BMC Res. Notes 2011, 4, 445. [CrossRef]

48. Singh, H.; Bharadvaja, N. Treasuring the computational approach in medicinal plant research. Prog. Biophys. Mol. Biol. 2021, 164, 19-32. [CrossRef]

49. Mitscher, L.A.; Baker, W. Tuberculosis: A search for novel therapy starting with natural products. Med. Res. Rev. 1998, 18, 363-374. [CrossRef]

50. Gautam, R.; Saklani, A.; Jachak, S.M. Indian medicinal plants as a source of antimycobacterial agents. J. Ethnopharmacol. 2007, 110, 200-234. [CrossRef]

51. Miryala, S.K.; Basu, S.; Naha, A.; Debroy, R.; Ramaiah, S.; Anbarasu, A.; Natarajan, S. Identification of bioactive natural compounds as efficient inhibitors against Mycobacterium tuberculosis protein-targets: A molecular docking and molecular dynamics simulation study. J. Mol. Liq. 2021, 341, 117340. [CrossRef]

52. Parthasarathy, R.; Sarma, G.; Janardhanam, B.; Ramachandran, P.; Santha, T.; Sivasubramanian, S.; Somasundaram, P.; Tripathy, S. Hepatic toxicity in south indian patients during treatment of tuberculosis with short-course regimens containing isoniazid, rifampicin and pyrazinamide. Tubercle 1986, 67, 99-108. [CrossRef]

53. Mohan, A.; Sharma, S.K. Side effects of antituberculosis drugs. Am. J. Respir. Crit. Care Med. 2004, 169, 882-883. [CrossRef]

54. Yew, W.W.; Leung, C.C. Antituberculosis drugs and hepatotoxicity. Respirology 2006, 11, 699-707. [CrossRef]

55. Goldberger, M.J. Antituberculosis Agents. Med. Clin. N. Am. 1988, 72, 661-668. [CrossRef]

56. Tostmann, A.; Boeree, M.J.; Aarnoutse, R.E.; De Lange, W.C.; van der Ven, A.J.; Dekhuijzen, R. Antituberculosis drug-induced hepatotoxicity: Concise up-to-date review. J. Gastroenterol. Hepatol. 2008, 23, 192-202. [CrossRef]

57. Yee, D.; Valiquette, C.; Pelletier, M.; Parisien, I.; Rocher, I.; Menzies, D. Incidence of Serious Side Effects from First-Line Antituberculosis Drugs among Patients Treated for Active Tuberculosis. Am. J. Respir. Crit. Care Med. 2003, 167, $1472-1477$. [CrossRef] [PubMed]

58. Jimenez-Arellanes, A.; Meckes, M.; Ramírez, R.; Torres, J.; Luna-Herrera, J. Activity against multidrug-resistantMycobacterium tuberculosis in Mexican plants used to treat respiratory diseases. Phytotherapy Res. 2003, 17, 903-908. [CrossRef] [PubMed]

59. Fauziyah, P.N.; Sukandar, E.Y.; Ayuningtyas, D.K. Combination Effect of Antituberculosis Drugs and Ethanolic Extract of Selected Medicinal Plants against Multi-Drug Resistant Mycobacterium tuberculosis Isolates. Sci. Pharm. 2017, 85, 14. [CrossRef] [PubMed]

60. Subramani, R.; Narayanasamy, M.; Feussner, K.-D. Plant-derived antimicrobials to fight against multi-drug-resistant human pathogens. 3 Biotech 2017, 7, 172. [CrossRef]

61. Jiménez-Arellanes, A.; Meckes, M.; Torres, J.; Luna-Herrera, J. Antimycobacterial triterpenoids from Lantana hispida (Verbenaceae). J. Ethnopharmacol. 2007, 111, 202-205. [CrossRef]

62. Erdemoglu, N.; Sener, B.; Palittapongarnpim, P. Antimycobacterial Activity ofTaxus baccata. Pharm. Biol. 2003, 41, 614-615. [CrossRef]

63. Ignacimuthu, S.; Shanmugam, N. Antimycobacterial activity of two natural alkaloids, vasicine acetate and 2-acetyl benzylamine, isolated from Indian shrub Adhatoda vasica Ness. leaves. J. Biosci. 2010, 35, 565-570. [CrossRef]

64. Madikizela, B.; Aderogba, M.; Finnie, J.; Van Staden, J. Isolation and characterization of antimicrobial compounds from Terminalia phanerophlebia Engl. \& Diels leaf extracts. J. Ethnopharmacol. 2014, 156, 228-234. [CrossRef]

65. Saravanakumar, A.; Ganesh, M.; Peng, M.M.; Aziz, A.S.; Jang, H.T. Comparative antioxidant and antimycobacterial activities ofOpuntia ficus-indicafruit extracts from summer and rainy seasons. Front. Life Sci. 2015, 8, 182-191. [CrossRef]

66. Mohamad, S.; Zin, N.M.; Wahab, H.A.; Ibrahim, P.; Sulaiman, S.F.; Zahariluddin, A.S.M.; Noor, S.S.M. Antituberculosis potential of some ethnobotanically selected Malaysian plants. J. Ethnopharmacol. 2011, 133, 1021-1026. [CrossRef]

67. Luo, X.; Pires, D.; Aínsa, J.A.; Gracia, B.; Duarte, N.; Mulhovo, S.; Anes, E.; Ferreira, M.-J.U. Zanthoxylum capense constituents with antimycobacterial activity against Mycobacterium tuberculosis in vitro and ex vivo within human macrophages. $J$. Ethnopharmacol. 2013, 146, 417-422. [CrossRef]

68. Gouveia-Figueira, S.C.; Gouveia, C.A.; Carvalho, M.J.; Rodrigues, A.I.P.C.; Nording, M.L.; Castilho, P.C. Antioxidant Capacity, Cytotoxicity and Antimycobacterial Activity of Madeira Archipelago Endemic Helichrysum Dietary and Medicinal Plants. Antioxidants 2014, 3, 713-729. [CrossRef] 
69. Jyoti, A.; Nam, K.-W.; Jang, W.S.; Kim, Y.-H.; Kim, S.-K.; Lee, B.-E.; Song, H.-Y. Antimycobacterial activity of methanolic plant extract of Artemisia capillaris containing ursolic acid and hydroquinone against Mycobacterium tuberculosis. J. Infect. Chemother. 2016, 22, 200-208. [CrossRef]

70. Fadipe, V.O.; Mongalo, N.I.; Opoku, A.R.; Dikhoba, P.M.; Makhafola, T.J. Isolation of anti-mycobacterial compounds from Curtisia dentata (Burm.f.) C.A.Sm (Curtisiaceae). BMC Complement. Altern. Med. 2017, 17, 306. [CrossRef]

71. León-Díaz, R.; Meckes, M.; Said-Fernández, S.; Molina-Salinas, G.M.; Vargas-Villarreal, J.; Torres, J.; Luna-Herrera, J.; JiménezArellanes, A. Antimycobacterial neolignans isolated from Aristolochia taliscana. Memórias Inst. Oswaldo Cruz 2010, $105,45-51$. [CrossRef]

72. Prabu, A.; Seenivasan, P.; Kumar, V. Antimycobacterial activity of certain mangrove plants against multi-drug resistant Mycobacterium tuberculosis. Asian J. Med. Sci. 2014, 5, 54-57. [CrossRef]

73. Kirimuhuzya, C.; Waako, P.; Joloba, M.; Odyek, O. The anti-mycobacterial activity of Lantana camara a plant traditionally used to treat symptoms of tuberculosis in South-western Uganda. Afr. Health Sci. 2009, 9, 40-45.

74. Nguta, J.M.; Appiah-Opong, R.; Nyarko, A.K.; Yeboah-Manu, D.; Addo, P.G.; Otchere, I.; Kissi-Twum, A. Antimycobacterial and cytotoxic activity of selected medicinal plant extracts. J. Ethnopharmacol. 2016, 182, 10-15. [CrossRef]

75. Gupta, P.; Bhatter, P.; D’Souza, D.; Tolani, M.; Daswani, P.; Tetali, P.; Birdi, T. Evaluating the anti Mycobacterium tuberculosis activity of Alpinia galanga (L.) Willd. axenically under reducing oxygen conditions and in intracellular assays. BMC Complement. Altern. Med. 2014, 14, 84. [CrossRef]

76. Nirmal, C.R.; Ebenezer, R.S.; Kannan, P.; Balasubramanian, M.; Thirunavukkarasu, I.; Mondal, R.; Dusthackeer, A. Antituberculosis activity of bio-active compounds from Lantana camara L., Euphorbia hirta L., Mukia maderaspatana (L.) M. Roem, and Abutilon indicum (L.). Eur. J. Integr. Med. 2020, 35, 101105. [CrossRef]

77. Martini, M.C.; Zhang, T.; Williams, J.T.; Abramovitch, R.B.; Weathers, P.J.; Shell, S.S. Artemisia annua and Artemisia afra extracts exhibit strong bactericidal activity against Mycobacterium tuberculosis. J. Ethnopharmacol. 2020, 262, 113191. [CrossRef] [PubMed]

78. Assam, J.P.A.; Tcham, M.F.Y.; Moni, N.E.D.F.; Betote, D.P.H.; Fossi, T.C.; Penlap, B.V. Phytochemical screening, Antimycobacterial activity of three medicinal Cameroonians plants and Acute toxicity of hydroethanolic extract of Vitellaria paradoxa. J. Drug Deliv. Ther. 2020, 10, 96-104. [CrossRef]

79. Molina-Salinas, G.M.; Uc-Cachón, A.H.; Peña-Rodríguez, L.M.; Beh, A.D.J.D.; Gracía-Medrano, R.M.E.; García-Medrano, R.M.E. Bactericidal Effect of the Leaf Extract from Musa spp. (AAB Group, Silk Subgroup), cv. "Manzano" Against Multidrug-Resistant Mycobacterium tuberculosis. J. Med. Food 2019, 22, 1183-1185. [CrossRef] [PubMed]

80. Sanchez-Chavez, A.C.; Salazar, A.; Zepeda-Vallejo, L.G.; Jesús, M.D.L.H.D.; Quintos-Escalante, M.; Vargas-Díaz, M.E.; LunaHerrera, J. Trixis angustifolia hexanic extract displays synergistic antibacterial activity against M. tuberculosis. Nat. Prod. Res. 2017, 33, 1477-1481. [CrossRef]

81. Hernández-García, E.; García, A.; Garza-González, E.; Alanís, F.G.A.; Rivas-Galindo, V.M.; Rodríguez-Rodríguez, J.; AlcantarRosales, V.M.; Delgadillo-Puga, C.; Camacho-Corona, M.D.R. Chemical composition of Acacia farnesiana (L) wild fruits and its activity against Mycobacterium tuberculosis and dysentery bacteria. J. Ethnopharmacol. 2019, 230, 74-80. [CrossRef]

82. Kahaliw, W.; Aseffa, A.; Abebe, M.; Teferi, M.; Engidawork, E. Evaluation of the antimycobacterial activity of crude extracts and solvent fractions of selected Ethiopian medicinal plants. BMC Complement. Altern. Med. 2017, 17, 143. [CrossRef]

83. Kaur, R.; Kaur, H. Antitubercular activity and phytochemical screening of selected medicinal plants. Orient. J. Chem. 2015, 31, 597-600. [CrossRef]

84. Rahgozar, N.; Khaniki, G.B.; Sardari, S. Evaluation of Antimycobacterial and Synergistic Activity of Plants Selected Based on Cheminformatic Parameters. Iran. Biomed. J. 2018, 22, 401-407. [CrossRef]

85. Sudjarwo, S.A.; Wardani, G.; Eraiko, K. The potency of Pinus merkusii extract nanoparticles as anti Mycobacterium tuber-culosis: An in vitro study. Int. J. Nutr. Pharmacol. Neurol. Dis. 2019, 9, 48. [CrossRef]

86. Bhagat, V.C.; Kondawar, M.S. Antitubercular Potential of Dendrophthoe Falcate (L.) and Tridax Procumbens (L.) Plants Extracts Against H37rv Stain of Mycobacteria Tuberculosis. Int. J. Pharmaceut. Sci. Res. 2019, 10, 51-259.

87. Tiam, E.R.; Bikobo, D.S.N.; Zintchem, A.A.A.; Ii, N.M.N.; Ndedi, E.D.F.M.; Diboué, P.H.B.; Nyegue, M.A.; Atchadé, A.D.T.; Pegnyemb, D.E.; Bochet, C.G.; et al. Secondary metabolites from Triclisia gilletii (De Wild) Staner (Menispermaceae) with antimycobacterial activity against Mycobacterium tuberculosis. Nat. Prod. Res. 2017, 33, 642-650. [CrossRef]

88. Komape, N.P.M.; Bagla, V.P.; Kabongo-Kayoka, P.; Masoko, P. Anti-mycobacteria potential and synergistic effects of combined crude extracts of selected medicinal plants used by Bapedi traditional healers to treat tuberculosis related symptoms in Limpopo Province, South Africa. BMC Complement. Altern. Med. 2017, 17, 128. [CrossRef]

89. Vaidya, S.; Sharma, J.; Maniar, J.; Prabhu, N.; Mamawala, M.; Joshi-Pundit, S.; Chowdhary, A. Assessment of anti-tuberculosis activity of extracts of cinnamomum verum and solanun surattense along with isoniazid. BMC Complement. Alt. Med. 2018, 18, 5. [CrossRef]

90. Mohamad, S.; Ismail, N.N.; Parumasivam, T.; Ibrahim, P.; Osman, H.; Wahab, H.A. Antituberculosis activity, phytochemical identification of Costus speciosus (J. Koenig) Sm., Cymbopogon citratus (DC. Ex Nees) Stapf. and Tabernaemontana coronaria (L.) Willd. and their effects on the growth kinetics and cellular integrity of Mycobacterium tuberculosis H37Rv. BMC Complement. Altern. Med. 2018, 18, 5. [CrossRef]

91. Jang, W.S.; Jyoti, A.; Kim, S.; Nam, K.-W.; Ha, T.K.Q.; Oh, W.K.; Song, H.-Y. In vitro antituberculosis activity of diterpenoids from the Vietnamese medicinal plant Croton tonkinensis. J. Nat. Med. 2015, 70, 127-132. [CrossRef] 
92. Choi, W.H.; Lee, I.A. The anti-tubercular activity of Melia azedarach L. and Lobelia chinensis Lour. and their potential as effective anti-Mycobacterium tuberculosis candidate agents. Asian Pac. J. Trop. Biomed. 2016, 6, 830-835. [CrossRef]

93. Das, N.; Jena, P.K.; Pradhan, S.K. Arabinosyltransferase C enzyme of Mycobacterium tuberculosis, a potential drug target: An insight from molecular docking study. Heliyon 2020, 6, e02693. [CrossRef]

94. Sundar, S.; Thangamani, L.; Manivel, G.; Kumar, P.; Piramanayagam, S. Molecular docking, molecular dynamics and MM/PBSA studies of FDA approved drugs for protein kinase a of Mycobacterium tuberculosis; application insights of drug repurposing. Inform. Med. Unlocked 2019, 16, 100210. [CrossRef]

95. Gising, J.; Nilsson, M.T.; Odell, L.R.; Yahiaoui, S.; Lindh, M.; Iyer, H.; Sinha, A.M.; Srinivasa, B.R.; Larhed, M.; Mowbray, S.L.; et al. Trisubstituted Imidazoles as Mycobacterium tuberculosis Glutamine Synthetase Inhibitors. J. Med. Chem. 2012, 55, $2894-2898$. [CrossRef]

96. Pearce, M.J.; Arora, P.; Festa, R.A.; Butler-Wu, S.M.; Gokhale, R.S.; Darwin, K.H. Identification of substrates of the Mycobacterium tuberculosis proteasome. EMBO J. 2006, 25, 5423-5432. [CrossRef]

97. Kumar, M.; Chung, S.-M.; Enkhtaivan, G.; Patel, R.; Shin, H.-S.; Mistry, B. Molecular Docking Studies and Biological Evaluation of Berberine-Benzothiazole Derivatives as an Anti-Influenza Agent via Blocking of Neuraminidase. Int. J. Mol. Sci. 2021, 22, 2368. [CrossRef]

98. Quintero-Fabian, S.; Ortuño-Sahagún, D.; Vázquez-Carrera, M.; López-Roa, R.I. Alliin, a Garlic (Allium sativum) Compound, Prevents LPS-Induced Inflammation in 3T3-L1 Adipocytes. Mediat. Inflamm. 2013, 2013, 381815. [CrossRef]

99. Zeng, Y.; Li, Y.; Yang, J.; Pu, X.; Du, J.; Yang, X.; Yang, T.; Yang, S. Therapeutic Role of Functional Components in Alliums for Preventive Chronic Disease in Human Being. Evidence-Based Complement. Altern. Med. 2017, 2017, 9402849. [CrossRef]

100. Bergfield, W.F. Final report of the cosmetic ingredient review. Int. J. Toxicol. 2007, 26, 1-50.

101. Groom, Q.J.; Reynolds, T. Barbaloin inAloeSpecies. Planta Med. 1987, 53, 345-348. [CrossRef]

102. Dinesh, M.; Roopan, S.M.; Selvaraj, C.I. Photocatalytic degradation of nitrophenol using biologically active Phyllanthus emblica seed extract. J. Photochem. Photobiol. B Biol. 2016, 161, 273-278. [CrossRef]

103. Phillips, O.A.; Udo, E.E.; Varghese, R. Antimycobacterial Activities of Novel 5-(1H-1,2,3-Triazolyl)Methyl Oxazolidinones. Tuberc. Res. Treat. 2012, 2012, 1-7. [CrossRef]

104. Molina-Salinas, G.M.; Ramos-Guerra, M.C.; Vargas-Villarreal, J.; Mata-Cárdenas, B.D.; Becerril-Montes, P.; Said-Fernández, S. Bactericidal Activity of Organic Extracts from Flourensia cernua DC against Strains of Mycobacterium tuberculosis. Arch. Med. Res. 2006, 37, 45-49. [CrossRef]

105. Prakash, P.; Kumari, N.; Gayathiri, E.; Selvam, K.; Ragunathan, M.G.; Chandrasekaran, M.; Al-Dosary, M.A.; Hatamleh, A.A.; Nadda, A.K.; Kumar, M. In Vitro and In Silico Toxicological Properties of Natural Antioxidant Therapeutic Agent Azima tetracantha. LAM. Antioxidants 2021, 10, 1307. [CrossRef]

106. Romano, J.D.; Tatonetti, N.P. Informatics and Computational Methods in Natural Product Drug Discovery: A Review and Perspectives. Front. Genet. 2019, 10, 368. [CrossRef]

107. Sharma, V.; Sarkar, I.N. Bioinformatics opportunities for identification and study of medicinal plants. Brief. Bioinform. 2013, 14, 238-250. [CrossRef] [PubMed]

108. Babar, M.; Zaidi, N.-U.-S.S.; Pothineni, V.R.; Ali, Z.; Faisal, S.; Hakeem, K.; Gul, A. Application of Bioinformatics and System Biology in Medicinal Plant Studies. In Springer Protocols Handbooks; Springer: Cham, Switzerland, 2017; pp. $375-393$. 